BUHEP-02-32

FERMILAB-Pub-02/267-T

hep-ph/0210299

\title{
The Collider Phenomenology of Technihadrons in the Technicolor Straw Man Model
}

\author{
Kenneth Lane ${ }^{1,2 *}$ and Stephen Mrenna ${ }^{2 \dagger}$ \\ ${ }^{1}$ Department of Physics, Boston University \\ 590 Commonwealth Avenue, Boston, Massachusetts 02215 \\ ${ }^{2}$ Fermi National Accelerator Laboratory \\ P.O. Box 500, Batavia, Illinois 60510
}

October 26, 2018

\begin{abstract}
We discuss the phenomenology of the lightest $S U(3)_{C}$ singlet and non-singlet technihadrons in the Straw Man Model of low-scale technicolor (TCSM). The technihadrons are assumed to be those arising in topcolor-assisted technicolor models in which topcolor is broken by technifermion condensates. We improve upon the description of the color-singlet sector presented in our earlier paper introducing the TCSM (hep-ph/9903369). These improvements are most important for subprocess energies well below the masses of the $\rho_{T}$ and $\omega_{T}$ vector technihadrons and, therefore, apply especially to $e^{+} e^{-}$colliders such as LEP and a low-energy linear collider. In the color-octet sector, we consider mixing of the gluon, the coloron $V_{8}$ from topcolor breaking, and four isosinglet color-octet technirho mesons $\rho_{T 8}$. We assume, as expected in walking technicolor, that these $\rho_{T 8}$ decay into $\bar{q} q, g g$, and $g \pi_{T}$ final states, but not into $\pi_{T} \pi_{T}$, where $\pi_{T}$ is a technipion. All the TCSM production and decay processes discussed here are included in the event generator PyтHIA. We present several simulations appropriate for the Tevatron Collider, and suggest benchmark model lines for further experimental investigation.
\end{abstract}

*lane@physics.bu.edu

†mrenna@fnal.gov 


\section{Overview of the TCSM}

In this paper we improve and extend the "technicolor straw man model" (TCSM) [1] of collider signatures for dynamical electroweak and flavor symmetry breaking. The TCSM is a simple phenomenology of the lowest-lying vector and pseudoscalar technihadrons expected in technicolor theories which have both a walking gauge coupling $\alpha_{T C}$ and topcolor interactions to generate the large mass of the top quark.

The main improvements arise in the color $S U(3)_{C}$ singlet sector of the model. The treatment in Ref. [1] assumed fermion-antifermion annihilations to technihadrons occurred at subprocess cm energy $\sqrt{\hat{s}} \sim M_{\rho_{T}, \omega_{T}}$. This is is appropriate in a hadron collider where the parton $\sqrt{\hat{s}}$ sweeps over the narrow TCSM resonances and is, therefore, strongly dominated by the poles. In this paper we modify the production amplitudes to make them accurate for $\sqrt{\hat{s}}$ below the resonances as well. These modifications involve adding the continuum amplitudes including, where appropriate, the three-point anomaly amplitudes for production of an electroweak gauge boson and a technipion $[2,3]$. This is particularly appropriate for technihadron searches at $e^{+} e^{-}$ colliders such as LEP $[4,5,6,7]$ (and the proposed linear collider if its early stage has $\sqrt{s} \lesssim 300 \mathrm{GeV}$ ).

Our extension of the TCSM includes several low-lying color-non-singlet, isosinglet states - four vector $\rho_{T 8}$ 's, the massive topcolor gauge boson, $V_{8}$, and a neutral pseudoscalar $\pi_{T 8}$. These particles are most readily produced and detected in hadron colliders - the Tevatron and the Large Hadron Collider (LHC)-because of their relatively large coupling to gluons. We have incorporated the improved and extended production processes in the event generator PYTHIA [8] to allow detailed investigation by collider experiments.

The modern description of technicolor $[9,10]$ requires a walking technicolor gauge coupling [11] to evade unwanted flavor-changing neutral currents and the assistance of topcolor interactions that are strong near $1 \mathrm{TeV}[12,13]$ to provide a large top quark mass. Both these elaborations on the basic technicolor/extended technicolor (TC/ETC) proposal require a large number $N_{D}$

of technifermion doublets. Many technifermions are needed to make the beta function of walking technicolor small. Many also seem required in topcolorassisted technicolor (TC2) to generate the hard masses of quarks and leptons, to induce the correct mixing between heavy and light quarks, and to break topcolor symmetry down to ordinary color (via technifermion condensation). 
A large number of technidoublets implies a small technipion decay constant $F_{T} \simeq F_{\pi} / \sqrt{N_{D}}$, where $F_{\pi}=246 \mathrm{GeV}$. This, in turn, implies a technicolor scale $\Lambda_{T C} \sim F_{T}$ and masses of a few hundred $\mathrm{GeV}$ for the lowest-lying technihadrons $\left(\pi_{T}, \rho_{T}, \omega_{T}\right)[14,15]$. The signatures of this low-scale technicolor have been sought in Run I of the Tevatron Collider [16, 17, 18, 19, 20]. In the past two years, several LEP experiments have published limits on color-singlet technihadrons $[5,6,7]$ which in some cases exceed those set at Fermilab. Run II will significantly extend the reach of Run I in color-singlet and octet sectors. If these low-scale technihadrons exist, they certainly will be seen in LHC experiments [21].

The TCSM provides a simple framework for these searches. First, and probably most important, we assume that the lowest-lying bound states of the lightest technifermions can be considered in isolation. The lightest technifermions are expected to be an isodoublet of color singlets, $\left(T_{U}, T_{D}\right)$. Color triplets, discussed below, will be heavier because of $S U(3)_{C}$ contributions to their hard (chiral symmetry breaking) masses. We assume that all technifermions transform under technicolor $S U\left(N_{T C}\right)$ as fundamentals. The electric charges of $\left(T_{U}, T_{D}\right)$ are $Q_{U}$ and $Q_{D}=Q_{U}-1$. The color-singlet bound states we consider are vector and pseudoscalar mesons. The vectors include a spin-one isotriplet $\rho_{T}^{ \pm, 0}$ and an isosinglet $\omega_{T}$. In topcolor-assisted technicolor, there is no need to invoke large isospin-violating extended technicolor interactions to explain the top-bottom splitting. Thus, techni-isospin can be a good approximate symmetry, so that $\rho_{T}$ and $\omega_{T}$ are nearly degenerate. Their mixing will be described in the neutral-sector propagator matrix, $\Delta_{0}$, in Eq. (21) below. The new, improved formulas for the decays and production of these technivector mesons will be presented in Sections 2 and 3.

The lightest pseudoscalar $\left(T_{U}, T_{D}\right)$ bound states, color-singlet technipions, also comprise an isotriplet $\Pi_{T}^{ \pm, 0}$ and an isosinglet $\Pi_{T}^{0 \prime}$. However, these are not mass eigenstates. Our second important assumption for the TCSM is that the isovectors are simple two-state mixtures of the longitudinal weak bosons $W_{L}^{ \pm}, Z_{L}^{0}$ - the true Goldstone bosons of dynamical electroweak symmetry breaking in the limit that the $S U(2) \otimes U(1)$ couplings $g, g^{\prime}$ vanish — and mass-eigenstate pseudo-Goldstone technipions $\pi_{T}^{ \pm}, \pi_{T}^{0}$ :

$$
\left|\Pi_{T}\right\rangle=\sin \chi\left|W_{L}\right\rangle+\cos \chi\left|\pi_{T}\right\rangle .
$$

We assume that $S U\left(N_{T C}\right)$ gauge interactions dominate the binding of all technifermions into technihadrons. Then the decay constants of color-singlet 
and nonsinglet $\pi_{T}$ are approximately equal, $F_{T} \simeq F_{\pi} / \sqrt{N_{D}}$, and the mixing angle is given by

$$
\sin \chi \simeq F_{T} / F_{\pi} \simeq 1 / \sqrt{N_{D}} \ll 1
$$

Similarly, $\left|\Pi_{T}^{0 \prime}\right\rangle=\cos \chi^{\prime}\left|\pi_{T}^{0 \prime}\right\rangle+\cdots$, where $\chi^{\prime}$ is another mixing angle and the ellipsis refer to other technipions needed to eliminate the two-technigluon anomaly from the $\Pi_{T}^{0 \prime}$ chiral current. It is unclear whether, like $\rho_{T}$ and $\omega_{T}$, these neutral technipions will be degenerate as we have previously proposed [15]. On one hand, they both contain the lightest $\bar{T} T$ as constituents. On the other, because of the anomaly cancellation, $\pi_{T}^{0 \prime}$ must contain other heavier technifermions such as the color triplets we discuss below. If $\pi_{T}^{0}$ and $\pi_{T}^{0 \prime}$ are nearly degenerate and if their widths are roughly equal, there may be appreciable $\pi_{T}^{0}-\pi_{T}^{0 \prime}$ mixing and, then, the lightest neutral technipions will be ideally-mixed $\bar{T}_{U} T_{U}$ and $\bar{T}_{D} T_{D}$ bound states. Searches for these technipions ought to consider both possibilities: they are nearly degenerate or not at all.

Color-singlet technipion decays are mediated by ETC and (in the case of $\left.\pi_{T}^{0 \prime}\right) S U(3)_{C}$ interactions. In the TCSM they are taken to be [22]:

$$
\begin{aligned}
\Gamma\left(\pi_{T} \rightarrow \bar{f}^{\prime} f\right) & =\frac{1}{16 \pi F_{T}^{2}} N_{f} p_{f} C_{1 f}^{2}\left(m_{f}+m_{f^{\prime}}\right)^{2} \\
\Gamma\left(\pi_{T}^{0 \prime} \rightarrow g g\right) & =\frac{1}{128 \pi^{3} F_{T}^{2}} \alpha_{C}^{2} C_{1 g}^{2} N_{T C}^{2} M_{\pi_{T}^{0 \prime}}^{\frac{3}{2}}
\end{aligned}
$$

Like elementary Higgs bosons, technipions are expected to couple to fermion mass. Thus, $C_{1 f}$ is an ETC-model dependent factor of order one except that TC2 suggests $\left|C_{1 t}\right| \lesssim m_{b} / m_{t}$ so that there is not a strong preference for technipions to decay to (or from) top quarks. The number of colors of fermion $f$ is $N_{f}$. The fermion momentum is $p_{f}$. The QCD coupling $\alpha_{C}$ is evaluated at $M_{\pi_{T}}$; and $C_{1 g}^{2}$ is a Clebsch of order one. The default values of these and other parameters are tabulated at the end of this paper. For $M_{\pi_{T}}<m_{t}-m_{b}$, these technipions are expected to decay as follows: $\pi_{T}^{+} \rightarrow c \bar{b}$ or $c \bar{s}$ or even $\tau^{+} \nu_{\tau} ; \pi_{T}^{0} \rightarrow b \bar{b}$ and, perhaps $c \bar{c}, \tau^{+} \tau^{-}$; and $\pi_{T}^{0 \prime} \rightarrow g g, b \bar{b}, c \bar{c}$, $\tau^{+} \tau^{-}$.

The breaking of topcolor to ordinary color is most economically achieved by color-triplet technifermions [23]. Therefore, we expect $S U(3)_{C}$-nonsinglet technihadrons to exist. The lightest of these may have masses not very much larger than their color-singlet counterparts. Production and detection of 
color-nonsinglet $\rho_{T}$ and $\pi_{T}$ have been studied before, both theoretically [24, $25]$ and experimentally $[18,19,20]$. In these studies it was assumed that there exists one doublet of color-triplet technifermions, one or more doublets of color-singlets, and their associated technihadrons. In the likely case that techni-isospin is a good symmetry, the most accessible states would then be an isosinglet $\rho_{T 8}$, produced as an $s$-channel resonance in $\bar{q} q$ and $g g$ collisions, and the technipions (as well as dijets) into which $\rho_{T 8}$ may decay. With the advent of TC2, this simple model became obsolete.

In TC2 models, the existence of a large $\bar{t} t$ (but not $\bar{b} b$ ) condensate and mass is due to $S U(3)_{1} \otimes U(1)_{1}$ gauge interactions which are strong near $1 \mathrm{TeV}$ [13]. The $S U(3)_{1}$ interaction is $t-b$ symmetric while $U(1)_{1}$ couplings are $t-b$ asymmetric. This makes these forces supercritical for the top quark, but subcritical for bottom. There are weaker $S U(3)_{2} \otimes U(1)_{2}$ gauge interactions in which light quarks (and leptons) may or may not participate.

The two $U(1)$ 's must be broken to weak hypercharge $U(1)_{Y}$ at an energy somewhat higher than $1 \mathrm{TeV}$ by electroweak-singlet condensates [23]. This breaking results in a heavy color-singlet $Z^{\prime}$ boson whose physics we do not consider in this paper (see, however, Refs. [26, 27]). The two $S U(3)$ 's are broken to their diagonal $S U(3)_{C}$ subgroup. When this happens, a degenerate octet of "colorons", $V_{8}$, mediate the broken topcolor $S U(3)$ interactions. There are two variants of TC2: The "standard" version [13], in which only the third generation quarks are $S U(3)_{1}$ triplets, and the "flavor-universal" version [28] in which all quarks are $S U(3)_{1}$ triplets. We consider both in this paper.

In either version of TC2, the $S U(3)$ 's are economically broken to $S U(3)_{C}$ subgroup by using technicolor and $U(1)_{1}$ interactions, both strong near $1 \mathrm{TeV}$. Following Ref. [23], we assume the existence of two electroweak doublets of technifermions, $T_{1}=\left(U_{1}, D_{1}\right)$ and $T_{2}=\left(U_{2}, D_{2}\right)$, which transform respectively as $\left(\mathbf{3}, \mathbf{1}, \mathbf{N}_{\mathbf{T C}}\right)$ and $\left(\mathbf{1}, \mathbf{3}, \mathbf{N}_{\mathbf{T C}}\right)$ under the two color groups and technicolor. The desired pattern of symmetry breaking occurs if $S U\left(N_{T C}\right)$ and $U(1)_{1}$ interactions work together to induce electroweak and $S U(3)_{1} \otimes S U(3)_{2}$ noninvariant condensates (which, of course, preserve $S U(3)_{C}$ and $U(1)_{E M}$ )

$$
\left\langle\bar{U}_{i L} U_{j R}\right\rangle=-W_{i j}^{U *} \Delta_{T}, \quad\left\langle\bar{D}_{i L} D_{j R}\right\rangle=-W_{i j}^{D *} \Delta_{T}, \quad(i, j=1,2) .
$$

Here, $W^{U}$ and $W^{D}$ are nondiagonal $U(2)$ matrices and $\Delta_{T} \sim \Lambda_{T C}^{3}$. Assuming the condensates are parity-conserving, $\left(W_{i j}^{U, D}\right)^{*}=W_{j i}^{U, D}$. Then, unitarity 
implies

$$
\begin{aligned}
& W_{11}^{U, D}+W_{22}^{U, D}=0, \\
& W_{12}^{U, D}=\left(W_{21}^{U, D}\right)^{*}=e^{i \phi_{U, D}} \sqrt{1-\left(W_{11}^{U, D}\right)^{2}},
\end{aligned}
$$

where $\phi_{U, D}$ are phases to be chosen.

This minimal TC2 scenario leads to a rich spectrum of color-nonsinglet states readily accessible in hadron collisions. We concentrate on the lowestlying isosinglet color octets. In addition to the colorons, $V_{8}$, these include four $\rho_{T 8}$ formed from $\bar{T}_{i} T_{j}$, and a technipion $\pi_{T 8}$. These states are constructed in Section 4. In Section 5 we discuss the decay rates of $\rho_{T 8}$ and $V_{8}$ under the simplifying assumption that $M_{\rho_{T 8}}<2 M_{\pi_{T}}$. As in the color-singlet TCSM, this is likely because the large coupling of walking technicolor significantly enhances the ETC contribution to $M_{\pi_{T}}$ [14]. In the extreme walking $\alpha_{T C}$ limit, $M_{\pi_{T}} \simeq M_{\rho_{T 8}}$. In Section 6 we present the dijet production cross sections, using the fully mixed propagator of gluons, colorons and the four $\rho_{T 8}$. These cross sections are now encoded in the PYTHIA event generator [8]. Simulated signals (at the parton level) appropriate to Run I of the Tevatron Collider are presented in Section 7 , with special attention to the effects on the $\bar{t} t$ invariant mass distribution.

Two comments are in order before moving on. First, it has been argued in Ref. [29] that $B_{d}-\bar{B}_{d}$ mixing constrains $M_{V_{8}} \tan \theta_{3}>1-2 \mathrm{TeV}$ in standard, but not flavor-universal, TC2 (also see Ref. [30]). Here, $\theta_{3}$ is the $S U(3)_{1}-$ $S U(3)_{2}$ mixing angle defined in Section 4 below. This constraint relies on the form of quark mass and mixing matrices expected in ETC/TC2. We view as independent and complementary the limit on $M_{V_{8}} \tan \theta_{3}$ that may be derived from collider jet data. Second, Bertram and Simmons have used D $\varnothing$ jet data from Run I to place the limit $M_{V_{8}} \tan \theta_{3}>0.84 \mathrm{TeV}$ in flavor-universal TC2 [31]. Their analysis did not include the potential complicating effects of $\rho_{T 8}$ and their interference with $V_{8}$. Our TCSM analysis closes this loophole. We find limits on the coloron mass as high or higher than theirs.

\section{Decays of Color-Singlet $\rho_{T}$ and $\omega_{T}$}

In the limit that the electroweak couplings $g, g^{\prime}=0$, the $\rho_{T}$ and $\omega_{T}$ decay as

$$
\rho_{T} \rightarrow \Pi_{T} \Pi_{T}=\cos ^{2} \chi\left(\pi_{T} \pi_{T}\right)+2 \sin \chi \cos \chi\left(W_{L} \pi_{T}\right)+\sin ^{2} \chi\left(W_{L} W_{L}\right) ;
$$




$$
\omega_{T} \rightarrow \Pi_{T} \Pi_{T} \Pi_{T}=\cos ^{3} \chi\left(\pi_{T} \pi_{T} \pi_{T}\right)+\cdots .
$$

The $\rho_{T}$ decay amplitude is

$$
\mathcal{M}\left(\rho_{T}(q) \rightarrow \pi_{A}\left(p_{1}\right) \pi_{B}\left(p_{2}\right)\right)=g_{\rho_{T}} \mathcal{C}_{A B} \epsilon(q) \cdot\left(p_{1}-p_{2}\right)
$$

where $\epsilon(q)$ is the $\rho_{T}$ polarization vector; $\alpha_{\rho_{T}} \equiv g_{\rho_{T}}^{2} / 4 \pi=2.91\left(3 / N_{T C}\right)$ is scaled naively from QCD and $N_{T C}=4$ is used in calculations; and

$$
\mathcal{C}_{A B}= \begin{cases}\sin ^{2} \chi & \text { for } W_{L}^{+} W_{L}^{-} \text {or } W_{L}^{ \pm} Z_{L}^{0} \\ \sin \chi \cos \chi & \text { for } W_{L}^{+} \pi_{T}^{-}, W_{L}^{-} \pi_{T}^{+} \text {or } W_{L}^{ \pm} \pi_{T}^{0}, Z_{L}^{0} \pi_{T}^{ \pm} \\ \cos ^{2} \chi & \text { for } \pi_{T}^{+} \pi_{T}^{-} \text {or } \pi_{T}^{ \pm} \pi_{T}^{0} .\end{cases}
$$

The $\rho_{T}$ decay rate to two technipions is then (for later use in cross sections, we quote the energy-dependent width for a $\rho_{T}$ mass of $\sqrt{\hat{s}}$ )

$$
\Gamma\left(\rho_{T}^{0} \rightarrow \pi_{A}^{+} \pi_{B}^{-}\right)=\Gamma\left(\rho_{T}^{ \pm} \rightarrow \pi_{A}^{ \pm} \pi_{B}^{0}\right)=\frac{2 \alpha_{\rho_{T}} \mathcal{C}_{A B}^{2}}{3} \frac{p^{3}}{\hat{s}}
$$

where $p=\left[\left(\hat{s}-\left(M_{A}+M_{B}\right)^{2}\right)\left(\hat{s}-\left(M_{A}-M_{B}\right)^{2}\right)\right]^{\frac{1}{2}} / 2 \sqrt{\hat{s}}$ is the $\pi_{T}$ momentum in the $\rho_{T}$ rest frame.

Walking technicolor enhancements of technipion masses are likely to close off the channels $\rho_{T} \rightarrow \pi_{T} \pi_{T}, \omega_{T} \rightarrow \pi_{T} \pi_{T} \pi_{T}$ and even the isospin-violating $\omega_{T} \rightarrow \pi_{T} \pi_{T}$ [14]. A $\rho_{T}^{0}$ of mass $200 \mathrm{GeV}$, say, may then decay to $W_{L} \pi_{T}$ or $W_{L} W_{L}$, but such $\omega_{T}$ decays are strongly suppressed (see Eq. (15 below). Therefore, all $\omega_{T}$ decays are electroweak: $\omega_{T} \rightarrow \gamma \pi_{T}^{0}, Z^{0} \pi_{T}^{0}, W^{ \pm} \pi_{T}^{\mp}$, and $\bar{f} f$. Here, $Z$ and $W$ are transversely polarized. ${ }^{1}$ Furthermore, since we expect $\sin ^{2} \chi \ll 1$, the electroweak decays of $\rho_{T}$ to the transverse gauge bosons $\gamma, W, Z$ plus a technipion may be competitive with the open-channel strong decays.

As discussed in Ref. [1], the amplitude for $V_{T}=\rho_{T}, \omega_{T}$ decay to any transversely polarized electroweak boson $G$ plus a technipion is given by

$$
\begin{aligned}
\mathcal{M}\left(V_{T}(q) \rightarrow G\left(p_{1}\right) \pi_{T}\left(p_{2}\right)\right) & =\frac{e V_{V_{T} G \pi_{T}}}{M_{V}} \epsilon^{\mu \nu \lambda \rho} \epsilon_{\mu}(q) \epsilon_{\nu}^{*}\left(p_{1}\right) q_{\lambda} p_{1 \rho} \\
& +\frac{e A_{V_{T} G \pi_{T}}}{M_{A}}\left(\epsilon(q) \cdot \epsilon^{*}\left(p_{1}\right) q \cdot p_{1}-\epsilon(q) \cdot p_{1} \epsilon^{*}\left(p_{1}\right) \cdot q\right) .
\end{aligned}
$$

\footnotetext{
${ }^{1}$ Strictly speaking, the identification of $W$ and $Z$ decay products as longitudinal or transverse is approximate, becoming exact in the limit of very large $M_{\rho_{T}, \omega_{T}}$.
} 
The first term corresponds to the vector coupling of $G$ to the constituent technifermions of $V_{T}$ and $\pi_{T}$ and the second term to its axial-vector coupling. The technicolor-scale parameter $M_{V}$ is expected to be of order several $100 \mathrm{GeV} .{ }^{2}$ The mass parameter $M_{A}$ is expected to be comparable to $M_{V}$. Note that the amplitudes for emission of longitudinally polarized bosons in Eq. (7) and transversely polarized ones in Eq. (10) are noninterfering, as they should be. Adopting a "valence technifermion" model for the graphs describing Eq. (10) - a model which works very well for $\omega, \rho \rightarrow \gamma \pi$ and $\gamma \eta$ in QCD - CP-invariance implies that the $V$ and $A$ coefficients in this amplitude are given in our normalization by ${ }^{3}$

$$
V_{V_{T} G \pi_{T}}=2 \operatorname{Tr}\left(Q_{V_{T}}\left\{Q_{G_{V}}^{\dagger}, Q_{\pi_{T}}^{\dagger}\right\}\right), \quad A_{V_{T} G \pi_{T}}=2 \operatorname{Tr}\left(Q_{V_{T}}\left[Q_{G_{A}}^{\dagger}, Q_{\pi_{T}}^{\dagger}\right]\right) .
$$

In the TCSM, with electric charges $Q_{U}, Q_{D}$ for $T_{U}, T_{D}$, the generators $Q$ in Eq. (11) are given by

$$
\begin{aligned}
& Q_{\omega_{T}}=\left(\begin{array}{cc}
\frac{1}{2} & 0 \\
0 & \frac{1}{2}
\end{array}\right) \\
& Q_{\rho_{T}^{0}}=\left(\begin{array}{cc}
\frac{1}{2} & 0 \\
0 & -\frac{1}{2}
\end{array}\right) ; \quad Q_{\rho_{T}^{+}}=Q_{\rho_{T}^{-}}^{\dagger}=\frac{1}{\sqrt{2}}\left(\begin{array}{cc}
0 & 1 \\
0 & 0
\end{array}\right) \\
& Q_{\pi_{T}^{0}}=\cos \chi\left(\begin{array}{cc}
\frac{1}{2} & 0 \\
0 & -\frac{1}{2}
\end{array}\right) ; \quad Q_{\pi_{T}^{+}}=Q_{\pi_{T}^{-}}^{\dagger}=\frac{\cos \chi}{\sqrt{2}}\left(\begin{array}{cc}
0 & 1 \\
0 & 0
\end{array}\right) \\
& Q_{\pi_{T}^{0 \prime}}=\cos \chi^{\prime}\left(\begin{array}{cc}
\frac{1}{2} & 0 \\
0 & \frac{1}{2}
\end{array}\right) \\
& Q_{\gamma_{V}}=\left(\begin{array}{cc}
Q_{U} & 0 \\
0 & Q_{D}
\end{array}\right) ; \quad Q_{\gamma_{A}}=0 \\
& Q_{Z_{V}}=\frac{1}{\sin \theta_{W} \cos \theta_{W}}\left(\begin{array}{cc}
\frac{1}{4}-Q_{U} \sin ^{2} \theta_{W} & 0 \\
0 & -\frac{1}{4}-Q_{D} \sin ^{2} \theta_{W}
\end{array}\right) \\
& Q_{Z_{A}}=\frac{1}{\sin \theta_{W} \cos \theta_{W}}\left(\begin{array}{cc}
-\frac{1}{4} & 0 \\
0 & \frac{1}{4}
\end{array}\right)
\end{aligned}
$$

${ }^{2}$ The corresponding $\rho \rightarrow \gamma \pi$ parameter in QCD is about $400 \mathrm{MeV}$. A large $-N_{C}$ argument implies $M_{V} \simeq\left(F_{T} / f_{\pi}\right) 400 \mathrm{MeV} \simeq 350 \mathrm{GeV}$.

${ }^{3}$ We have neglected decays such as $\rho_{T}^{0} \rightarrow W_{T} W_{L}$ and $\rho_{T}^{0} \rightarrow W_{T} W_{T}$. The rate for the former is suppressed by $\tan ^{2} \chi$ relative to the rate for $\rho_{T}^{0} \rightarrow W_{T} \pi_{T}$ while the latter's rate is suppressed by $\alpha$. 


\begin{tabular}{|c|c|c|c|}
\hline Process & $V_{V_{T} G \pi_{T}}$ & $A_{V_{T} G \pi_{T}}$ & $\Gamma\left(V_{T} \rightarrow G \pi_{T}\right)$ \\
\hline \hline$\omega_{T} \rightarrow \gamma \pi_{T}^{0}$ & $c_{\chi}$ & 0 & $0.115 c_{\chi}^{2}$ \\
$\rightarrow \gamma \pi_{T}^{0 \prime}$ & $\left(Q_{U}+Q_{D}\right) c_{\chi^{\prime}}$ & 0 & $0.320 c_{\chi^{\prime}}^{2}$ \\
$\rightarrow Z^{0} \pi_{T}^{0}$ & $c_{\chi} \cot 2 \theta_{W}$ & 0 & $2.9 \times 10^{-3} c_{\chi}^{2}$ \\
$\rightarrow Z^{0} \pi_{T}^{0 \prime}$ & $-\left(Q_{U}+Q_{D}\right) c_{\chi^{\prime}} \tan \theta_{W}$ & 0 & $5.9 \times 10^{-3} c_{\chi^{\prime}}^{2}$ \\
$\rightarrow W^{ \pm} \pi_{T}^{\mp}$ & $c_{\chi} /\left(2 \sin \theta_{W}\right)$ & 0 & $2.4 \times 10^{-2} c_{\chi}^{2}$ \\
\hline$\rho_{T}^{0} \rightarrow \gamma \pi_{T}^{0}$ & $\left(Q_{U}+Q_{D}\right) c_{\chi}$ & 0 & $0.320 c_{\chi}^{2}$ \\
$\rightarrow \gamma \pi_{T}^{0 \prime}$ & $c_{\chi^{\prime}}$ & 0 & $0.115 c_{\chi}^{2}$ \\
$\rightarrow Z^{0} \pi_{T}^{0}$ & $-\left(Q_{U}+Q_{D}\right) c_{\chi} \tan \theta_{W}$ & 0 & $5.9 \times 10^{-3} c_{\chi}^{2}$ \\
$\rightarrow Z^{0} \pi_{T}^{0 \prime}$ & $c_{\chi^{\prime}} \cot 2 \theta_{W}$ & 0 & $2.9 \times 10^{-3} c_{\chi^{\prime}}^{2}$ \\
$\rightarrow W^{ \pm} \pi_{T}^{\mp}$ & 0 & $\pm c_{\chi} /\left(2 \sin \theta_{W}\right)$ & $0.143 c_{\chi}^{2}$ \\
\hline$\rho_{T}^{ \pm} \rightarrow \gamma \pi_{T}^{ \pm}$ & $\left(Q_{U}+Q_{D}\right) c_{\chi}$ & 0 & $0.320 c_{\chi}^{2}$ \\
$\rightarrow Z^{0} \pi_{T}^{ \pm}$ & $-\left(Q_{U}+Q_{D}\right) c_{\chi} \tan \theta_{W}$ & $\pm c_{\chi} / \sin 2 \theta_{W}$ & $0.153 c_{\chi}^{2}$ \\
$\rightarrow W^{ \pm} \pi_{T}^{0}$ & 0 & $\mp c_{\chi} /\left(2 \sin \theta_{W}\right)$ & $0.143 c_{\chi}^{2}$ \\
$\rightarrow W^{ \pm} \pi_{T}^{0 \prime}$ & $c_{\chi^{\prime}} /\left(2 \sin \theta_{W}\right)$ & 0 & $2.4 \times 10^{-2} c_{\chi^{\prime}}^{2}$ \\
\hline \hline
\end{tabular}

Table 1: Amplitudes and sample decay rates (in $\mathrm{GeV}$ ) for $V_{T} \rightarrow G \pi_{T}$. In the rate calculations, $M_{V_{T}}=210 \mathrm{GeV}, M_{\pi_{T}}=110 \mathrm{GeV}, M_{V}=M_{A}=100 \mathrm{GeV}$; technifermion charges are $Q_{U}+Q_{D}=\frac{5}{3} ; c_{\chi}=\cos \chi$ and $c_{\chi^{\prime}}=\cos \chi^{\prime}$; $G_{V}$ and $G_{A}$ refer to decays involving the vector and axial-vector couplings, respectively. Rates involving both couplings are summed in the last column.

$$
Q_{W_{V}^{+}}=Q_{W_{V}^{-}}^{\dagger}=-Q_{W_{A}^{+}}=-Q_{W_{A}^{-}}^{\dagger}=\frac{1}{2 \sqrt{2} \sin \theta_{W}}\left(\begin{array}{cc}
0 & 1 \\
0 & 0
\end{array}\right) .
$$

The decay rate for $V_{T} \rightarrow G \pi_{T}$ is

$$
\Gamma\left(V_{T} \rightarrow G \pi_{T}\right)=\frac{\alpha V_{V_{T} G \pi_{T}}^{2} p^{3}}{3 M_{V}^{2}}+\frac{\alpha A_{V_{T} G \pi_{T}}^{2} p\left(3 M_{G}^{2}+2 p^{2}\right)}{6 M_{A}^{2}},
$$

where $p$ is the $G$-momentum in the $V_{T}$ rest frame. The $V$ and $A$ coefficients and sample decay rates are listed in Table 1 . These are to be compared with the rates for decay into longitudinal $W$ and $Z$ bosons plus a technipion. For $M_{\rho_{T}}=210 \mathrm{GeV}, M_{\pi_{T}}=110 \mathrm{GeV}$, and $N_{T C}=4$, they are

$$
\begin{aligned}
\Gamma\left(\rho_{T}^{0} \rightarrow W_{L}^{ \pm} \pi_{T}^{\mp}\right) & =\Gamma\left(\rho_{T}^{ \pm} \rightarrow W_{L}^{ \pm} \pi_{T}^{0}\right)=2.78 \sin ^{2} \chi \cos ^{2} \chi \\
\Gamma\left(\rho_{T}^{ \pm} \rightarrow Z_{L}^{0} \pi_{T}^{\mp}\right) & =0.89 \sin ^{2} \chi \cos ^{2} \chi .
\end{aligned}
$$


For $\sin ^{2} \chi=1 / 9$, our nominal choice, and for $M_{V}=M_{A}=100 \mathrm{GeV}$, the rates for $\rho_{T}$ and $\omega_{T} \rightarrow \gamma \pi_{T}$ and for $\rho_{T} \rightarrow W_{T} \pi_{T}, Z_{T} \pi_{T}$ via axial vector coupling are comparable to these. Obviously, these transverse boson decay rates fall quickly for greater $M_{V}$ and $M_{A}$.

The rate for the isospin-violating decay $\omega_{T} \rightarrow W_{L}^{ \pm} \pi_{T}^{\mp}$ can be estimated as

$$
\Gamma\left(\omega_{T} \rightarrow W_{L}^{ \pm} \pi_{T}^{\mp}\right)=\left|\epsilon_{\rho \omega}\right|^{2} \Gamma\left(\rho_{T}^{0} \rightarrow W_{L}^{ \pm} \pi_{T}^{\mp}\right),
$$

where $\epsilon_{\rho \omega}$ is the $\rho_{T}-\omega_{T}$ mixing amplitude. In QCD, $\left|\epsilon_{\rho \omega}\right| \simeq 0.05$, so we expect this decay mode to be entirely negligible.

Finally, for completeness, we record again the decay rates for $\rho_{T}, \omega_{T} \rightarrow$ $f \bar{f}$. The $\rho_{T}$ decay rates to fermions with $N_{f}=1$ or 3 colors are

$$
\begin{aligned}
\Gamma\left(\rho_{T}^{0} \rightarrow f_{i} \bar{f}_{i}\right) & =\frac{N_{f} \alpha^{2} p}{3 \alpha_{\rho_{T}} \hat{s}}\left(\left(\hat{s}-m_{i}^{2}\right) A_{i}^{0}(\hat{s})+6 m_{i}^{2} \mathcal{R} e\left(\mathcal{A}_{i L}(\hat{s}) \mathcal{A}_{i R}^{*}(\hat{s})\right)\right), \\
\Gamma\left(\rho_{T}^{+} \rightarrow f_{i} \bar{f}_{i}^{\prime}\right) & =\frac{N_{f} \alpha^{2} p}{6 \alpha_{\rho_{T}} \hat{s}^{2}}\left(2 \hat{s}^{2}-\hat{s}\left(m_{i}^{2}+m_{i}^{\prime 2}\right)-\left(m_{i}^{2}-m_{i}^{\prime 2}\right)^{2}\right) A_{i}^{+}(\hat{s}),
\end{aligned}
$$

where a unit CKM matrix is assumed in the second equality. The quantities $A_{i}$ are given by

$$
\begin{aligned}
A_{i}^{ \pm}(\hat{s}) & =\frac{1}{8 \sin ^{4} \theta_{W}}\left|\frac{\hat{s}}{\hat{s}-\mathcal{M}_{W}^{2}}\right|^{2}, \\
A_{i}^{0}(\hat{s}) & =\left|\mathcal{A}_{i L}(\hat{s})\right|^{2}+\left|\mathcal{A}_{i R}(\hat{s})\right|^{2},
\end{aligned}
$$

where, for $\lambda=L, R$,

$$
\begin{aligned}
\mathcal{A}_{i \lambda}(\hat{s}) & =Q_{i}+\frac{2 \zeta_{i \lambda} \cot 2 \theta_{W}}{\sin 2 \theta_{W}}\left(\frac{\hat{s}}{\hat{s}-\mathcal{M}_{Z}^{2}}\right), \\
\zeta_{i L} & =T_{3 i}-Q_{i} \sin ^{2} \theta_{W}, \\
\zeta_{i R} & =-Q_{i} \sin ^{2} \theta_{W} .
\end{aligned}
$$

Here, $Q_{i}$ and $T_{3 i}= \pm 1 / 2$ are the electric charge and left-handed weak isospin of fermion $f_{i}$. Also, $\mathcal{M}_{W, Z}^{2}=M_{W, Z}^{2}-i \sqrt{\hat{s}} \Gamma_{W, Z}(\hat{s})$, where $\Gamma_{W, Z}(\hat{s})$ is the weak boson's energy-dependent width. ${ }^{4}$

\footnotetext{
${ }^{4}$ Note, for example, that $\Gamma_{Z}(\hat{s})$ includes a $t \bar{t}$ contribution when $\hat{s}>4 m_{t}^{2}$.
} 
The $\omega_{T}$ decay rates to fermions with $N_{f}$ colors are given by

$$
\Gamma\left(\omega_{T} \rightarrow \bar{f}_{i} f_{i}\right)=\frac{N_{f} \alpha^{2} p}{3 \alpha_{\rho_{T}} \hat{s}}\left(\left(\hat{s}-m_{i}^{2}\right) B_{i}^{0}(\hat{s})+6 m_{i}^{2} \mathcal{R} e\left(\mathcal{B}_{i L}(\hat{s}) \mathcal{B}_{i R}^{*}(\hat{s})\right)\right)
$$

where

$$
\begin{aligned}
& B_{i}^{0}(\hat{s})=\left|\mathcal{B}_{i L}(\hat{s})\right|^{2}+\left|\mathcal{B}_{i R}(\hat{s})\right|^{2} \\
& \mathcal{B}_{i \lambda}(\hat{s})=\left[Q_{i}-\frac{4 \zeta_{i \lambda} \sin ^{2} \theta_{W}}{\sin ^{2} 2 \theta_{W}}\left(\frac{\hat{s}}{\hat{s}-\mathcal{M}_{Z}^{2}}\right)\right]\left(Q_{U}+Q_{D}\right) .
\end{aligned}
$$

\section{Color-Singlet Technihadron Production Rates}

In this section we list the cross sections for the hadron collider subprocesses $q \bar{q} \rightarrow V_{T} \rightarrow \pi_{T} \pi_{T}, G \pi_{T}$, and $f \bar{f}$. The $e^{-} e^{+}$cross sections are obtained from the corresponding $q_{i} \bar{q}_{i}$ ones by multiplying the latter by 3 . To include $\rho_{T}-\omega_{T}$ interference in these cross sections, we use the mixed $\gamma-Z^{0}-\rho_{T}^{0}-\omega_{T}$ propagator matrix $\Delta_{0}$. It is the inverse of ${ }^{5}$

$$
\Delta_{0}^{-1}(s)=\left(\begin{array}{cccc}
s & 0 & s f_{\gamma \rho_{T}} & s f_{\gamma \omega_{T}} \\
0 & s-\mathcal{M}_{Z}^{2} & s f_{Z \rho_{T}} & s f_{Z \omega_{T}} \\
s f_{\gamma \rho_{T}} & s f_{Z \rho_{T}} & s-\mathcal{M}_{\rho_{T}^{0}}^{2} & 0 \\
s f_{\gamma \omega_{T}} & s f_{Z \omega_{T}} & 0 & s-\mathcal{M}_{\omega_{T}}^{2}
\end{array}\right) .
$$

To guarantee a photon pole at $s=0$, we assume only kinetic mixing between the gauge bosons and technivector mesons. In setting the off-diagonal $\rho_{T}^{0}$ $\omega_{T}$ elements of this matrix equal zero, we are guided by the smallness of this mixing in QCD and by the desire to keep the number of adjustable parameters in the TCSM small. This mixing can always be added if desired. The properly normalized $G V_{T}$ couplings are

$$
f_{G V_{T}}=2 \xi \operatorname{Tr}\left(Q_{G_{V}} Q_{V_{T}}^{\dagger}\right)
$$

\footnotetext{
${ }^{5}$ Note sign changes in the off-diagonal elements relative to the propagator matrices in Ref. [1].
} 
in particular, $f_{\gamma \rho_{T}}=\xi, f_{\gamma \omega_{T}}=\xi\left(Q_{U}+Q_{D}\right), f_{Z \rho_{T}}=\xi \cot 2 \theta_{W}$, and $f_{Z \omega_{T}}=$ $-\xi\left(Q_{U}+Q_{D}\right) \tan \theta_{W}$, where $\xi=\sqrt{\alpha / \alpha_{\rho_{T}}}$. In the charged sector, the $W^{ \pm}-\rho_{T}^{ \pm}$ matrix is the inverse of

$$
\Delta_{ \pm}^{-1}(s)=\left(\begin{array}{cc}
s-\mathcal{M}_{W}^{2} & s f_{W \rho_{T}} \\
s f_{W \rho_{T}} & s-\mathcal{M}_{\rho_{T}^{ \pm}}^{2}
\end{array}\right)
$$

where $f_{W \rho_{T}}=\xi /\left(2 \sin \theta_{W}\right)$.

The rates for production of any technipion pair, $\pi_{A} \pi_{B}=W_{L} W_{L}, W_{L} \pi_{T}$, and $\pi_{T} \pi_{T}$, in the isovector $\left(\rho_{T}\right)$ channel are: ${ }^{6}$

$$
\begin{aligned}
& \frac{d \hat{\sigma}\left(q_{i} \bar{q}_{i} \rightarrow \pi_{A}^{+} \pi_{B}^{-}\right)}{d \hat{t}} \\
& =\frac{\pi \alpha^{2} \mathcal{C}_{A B}^{2}\left(4 \hat{s} p^{2}-(\hat{t}-\hat{u})^{2}\right)}{12 \hat{s}^{2}} \sum_{\lambda=L, R} \mid Q_{i}\left(\Delta_{\gamma \gamma}(\hat{s})+f_{\gamma \rho_{T}}^{-1} \Delta_{\gamma \rho_{T}}\right) \\
& +\frac{2 \zeta_{i \lambda} \cot 2 \theta_{W}}{\sin 2 \theta_{W}}\left(\Delta_{Z Z}(\hat{s})+f_{Z \rho_{T}}^{-1} \Delta_{Z \rho_{T}}(\hat{s})\right)+\left.\left(\frac{Q_{i} \cos 2 \theta_{W}+2 \zeta_{i \lambda}}{\sin 2 \theta_{W}}\right) \Delta_{\gamma Z}\right|^{2},
\end{aligned}
$$

and

$$
\begin{aligned}
\frac{d \hat{\sigma}\left(u_{i} \bar{d}_{i} \rightarrow \pi_{A}^{+} \pi_{B}^{0}\right)}{d \hat{t}}= & \frac{\pi \alpha^{2} \mathcal{C}_{A B}^{2}\left(4 \hat{s} p^{2}-(\hat{t}-\hat{u})^{2}\right)}{96 \sin ^{4} \theta_{W} \hat{s}^{2}} \\
& \times\left|\Delta_{W W}(\hat{s})+f_{W \rho_{T}}^{-1} \Delta_{W \rho_{T}}(\hat{s})\right|^{2},
\end{aligned}
$$

where $p=\left[\left(\hat{s}-\left(M_{A}+M_{B}\right)^{2}\right)\left(\hat{s}-\left(M_{A}-M_{B}\right)^{2}\right)\right]^{\frac{1}{2}} / 2 \sqrt{\hat{s}}$ is the $\hat{s}$-dependent momentum of $\pi_{A, B}$. As usual, $\hat{t}=M_{A}^{2}-\sqrt{\hat{s}}\left(E_{A}-p \cos \theta\right), \hat{u}=M_{A}^{2}-$ $\sqrt{\hat{s}}\left(E_{A}+p \cos \theta\right)$, where $\theta$ is the c.m. production angle of $\pi_{A}$. The factor $4 \hat{s} p^{2}-(\hat{t}-\hat{u})^{2}=4 \hat{s} p^{2} \sin ^{2} \theta$. Because the $\rho_{T}-\omega_{T}$ mixing parameter $\epsilon_{\rho \omega}$ is expected to be very small, the rates for $q_{i} \bar{q}_{i} \rightarrow \omega_{T} \rightarrow \pi_{A}^{+} \pi_{B}^{-}$are ignored here.

The production of a transverse gauge boson plus technipion, $G \pi_{T}$, is dominated by the $\rho_{T}, \omega_{T}$ poles. However, in the limit $M_{\rho_{T}, \omega_{T}} \rightarrow \infty$, it still

\footnotetext{
${ }^{6}$ For $W^{+} W^{-}$and $W^{ \pm} Z^{0}$ production, one should add the standard model $t$ and $u^{-}$ channel amplitudes to the technicolor-modified $s$-channel amplitude. This is a small correction for a narrow $\rho_{T}$ when one effectively is always near the pole, as in a hadron collider. For a lepton collider far below the $\rho_{T}$ pole, these contributions are important. Similarly, the terms involving $\Delta_{\gamma \gamma}, \Delta_{Z Z}$ and $\Delta_{\gamma Z}$ in Eq. (24) are important only far below the pole. They are relevant for LEP, but not the Tevatron.
} 
proceeds via the usual axial vector anomaly process, $G_{1} \rightarrow G_{2} \pi_{T}$ [2]. This contribution interferes only with the $V_{V_{T} G_{2} \pi_{T}}$ term in Eq. (10). It is small in a hadron collider, but may be nonnegligible in an $e^{+} e^{-}$collider (such as LEP) operating well below the resonances. As discussed in Ref. [3], we include the anomaly contribution by simply adding it to the corresponding technivector amplitude. The cross section in the neutral channel is given by

$$
\begin{aligned}
& \frac{d \hat{\sigma}\left(q_{i} \bar{q}_{i} \rightarrow G \pi_{T}\right)}{d \hat{t}} \\
& =\frac{\pi \alpha^{2}}{24 \hat{s}}\left\{\left(\left|\mathcal{G}_{i L}^{V G \pi_{T}}(\hat{s})\right|^{2}+\left|\mathcal{G}_{i R}^{V G \pi_{T}}(\hat{s})\right|^{2}\right)\left(\hat{t}^{2}+\hat{u}^{2}-2 M_{G}^{2} M_{\pi_{T}}^{2}\right)\right. \\
& \left.\quad+\left(\left|\mathcal{G}_{i L}^{A G \pi_{T}}(\hat{s})\right|^{2}+\left|\mathcal{G}_{i R}^{A G \pi_{T}}(\hat{s})\right|^{2}\right)\left(\hat{t}^{2}+\hat{u}^{2}-2 M_{G}^{2} M_{\pi_{T}}^{2}+4 \hat{s} M_{G}^{2}\right)\right\},
\end{aligned}
$$

where, for quark helicities $\lambda=L, R$,

$$
\begin{aligned}
\mathcal{G}_{i \lambda}^{V G \pi_{T}}(\hat{s}) & =\sum_{V_{T}=\rho_{T}^{0}, \omega_{T}} \frac{V_{V_{T} G \pi_{T}}}{M_{V}}\left(Q_{i} \Delta_{\gamma V_{T}}(\hat{s})+\frac{2 \zeta_{i \lambda}}{\sin 2 \theta_{W}} \Delta_{Z V_{T}}(\hat{s})\right) \\
& +\frac{e N_{T C}}{8 \pi^{2} F_{T}}\left[V_{\gamma G \pi_{T}}\left(Q_{i} \Delta_{\gamma \gamma}+\frac{2 \zeta_{i \lambda}}{\sin 2 \theta_{W}} \Delta_{Z \gamma}\right)+V_{Z G \pi_{T}}\left(Q_{i} \Delta_{\gamma Z}+\frac{2 \zeta_{i \lambda}}{\sin 2 \theta_{W}} \Delta_{Z Z}\right)\right] ; \\
\mathcal{G}_{i \lambda}^{A G \pi_{T}}(\hat{s}) & =\sum_{V_{T}=\rho_{T}^{0}, \omega_{T}} \frac{A_{V_{T} G \pi_{T}}}{M_{A}}\left(Q_{i} \Delta_{\gamma V_{T}}(\hat{s})+\frac{2 \zeta_{i \lambda}}{\sin 2 \theta_{W}} \Delta_{Z V_{T}}(\hat{s})\right) .
\end{aligned}
$$

The anomaly factors in Eq. (27) are

$$
V_{G_{1} G_{2} \pi_{T}}=\operatorname{Tr}\left[Q_{\pi_{T}}^{\dagger}\left(\left\{Q_{G_{1}}, Q_{G_{2}}^{\dagger}\right\}_{L}+\left\{Q_{G_{1}}, Q_{G_{2}}^{\dagger}\right\}_{R}\right)\right],
$$

where $Q_{G_{R, L}}=Q_{G_{V}} \pm Q_{G_{A}}$ in Eq. (12). They are listed in Table 2. Note that $\hat{t}^{2}+\hat{u}^{2}-2 M_{G}^{2} M_{\pi_{T}}^{2}=2 \hat{s} p^{2}\left(1+\cos ^{2} \theta\right)$. The $G \pi_{T}$ cross section in the charged channel is given by (in the approximation of a unit CKM matrix)

$$
\begin{aligned}
\frac{d \hat{\sigma}\left(u_{i} \bar{d}_{i} \rightarrow G \pi_{T}\right)}{d \hat{t}} & =\frac{\pi \alpha^{2}}{48 \sin ^{2} \theta_{W} \hat{s}}\left\{\frac{A_{\rho_{T}^{+} G \pi_{T}}^{2}}{M_{A}^{2}}\left|\Delta_{W \rho_{T}}\right|^{2}\left(\hat{t}^{2}+\hat{u}^{2}-2 M_{G}^{2} M_{\pi_{T}}^{2}+4 \hat{s} M_{G}^{2}\right)\right. \\
& \left.+\left|\frac{e N_{T C}}{8 \pi^{2} F_{T}} V_{W+G \pi_{T}} \Delta_{W W}+\frac{V_{\rho_{T}^{+} G \pi_{T}}}{M_{V}} \Delta_{W \rho_{T}}\right|^{2}\left(\hat{t}^{2}+\hat{u}^{2}-2 M_{G}^{2} M_{\pi_{T}}^{2}\right)\right\} .
\end{aligned}
$$




\begin{tabular}{|c|c|}
\hline Process & $V_{G_{1} G_{2} \pi_{T}}$ \\
\hline \hline$\gamma \rightarrow \gamma \pi_{T}^{0}$ & $2\left(Q_{U}+Q_{D}\right) c_{\chi}$ \\
$\rightarrow \gamma \pi_{T}^{0 \prime}$ & $2\left(Q_{U}^{2}+Q_{D}^{2}\right) c_{\chi^{\prime}}$ \\
$\rightarrow Z^{0} \pi_{T}^{0}$ & $\left(Q_{U}+Q_{D}\right) c_{\chi}\left(1-4 \sin ^{2} \theta_{W}\right) / \sin 2 \theta_{W}$ \\
$\rightarrow Z^{0} \pi_{T}^{0 \prime}$ & $c_{\chi^{\prime}}\left[1-4\left(Q_{U}^{2}+Q_{D}^{2}\right) \sin ^{2} \theta_{W}\right] / \sin 2 \theta_{W}$ \\
$\rightarrow W^{ \pm} \pi_{T}^{\mp}$ & $\left(Q_{U}+Q_{D}\right) c_{\chi} /\left(2 \sin \theta_{W}\right)$ \\
\hline$Z^{0} \rightarrow \gamma \pi_{T}^{0}$ & $\left(Q_{U}+Q_{D}\right) c_{\chi}\left(1-4 \sin ^{2} \theta_{W}\right) / \sin 2 \theta_{W}$ \\
$\rightarrow \gamma \pi_{T}^{0 \prime}$ & $c_{\chi^{\prime}}\left[1-4\left(Q_{U}^{2}+Q_{D}^{2}\right) \sin ^{2} \theta_{W}\right] / \sin 2 \theta_{W}$ \\
$\rightarrow Z^{0} \pi_{T}^{0}$ & $-\left(Q_{U}+Q_{D}\right) c_{\chi} \cos 2 \theta_{W} / \cos ^{2} \theta_{W}$ \\
$\rightarrow Z^{0} \pi_{T}^{0 \prime}$ & $2 c_{\chi^{\prime}}\left[\cos 2 \theta_{W}+4\left(Q_{U}^{2}+Q_{D}^{2}\right) \sin \theta_{W}\right] / \sin ^{2} 2 \theta_{W}$ \\
$\rightarrow W^{ \pm} \pi_{T}^{\mp}$ & $-\left(Q_{U}+Q_{D}\right) c_{\chi} /\left(2 \cos \theta_{W}\right)$ \\
\hline$W^{ \pm} \rightarrow \gamma \pi_{T}^{ \pm}$ & $\left(Q_{U}+Q_{D}\right) c_{\chi} /\left(2 \sin \theta_{W}\right)$ \\
$\rightarrow Z^{0} \pi_{T}^{ \pm}$ & $-\left(Q_{U}+Q_{D}\right) c_{\chi} /\left(2 \cos \theta_{W}\right)$ \\
$\rightarrow W^{ \pm} \pi_{T}^{0}$ & 0 \\
$\rightarrow W^{ \pm} \pi_{T}^{0 \prime}$ & $c_{\chi^{\prime}} /\left(2 \sin ^{2} \theta_{W}\right)$ \\
\hline \hline
\end{tabular}

Table 2: Anomaly factors for $G_{1} \rightarrow G_{2} \pi_{T}$ for $G_{i}$ a transverse electroweak boson, $\gamma, Z^{0}, W^{ \pm}$. Here, $c_{\chi}=\cos \chi$ and $c_{\chi^{\prime}}=\cos \chi^{\prime}$.

The cross section for $q_{i} \bar{q}_{i} \rightarrow f_{j} \bar{f}_{j}$ in the color-singlet channel (with $m_{q_{i}}=0$ and allowing $m_{j} \neq 0$ for $t \bar{t}$ production) is

$$
\begin{aligned}
\frac{d \hat{\sigma}\left(q_{i} \bar{q}_{i} \rightarrow \gamma, Z \rightarrow \bar{f}_{j} f_{j}\right)}{d \hat{t}} & =\frac{N_{f} \pi \alpha^{2}}{3 \hat{s}^{2}}\left\{\left(\left(\hat{u}-m_{j}^{2}\right)^{2}+m_{j}^{2} \hat{s}\right)\left(\left|\mathcal{D}_{i j L L}\right|^{2}+\left|\mathcal{D}_{i j R R}\right|^{2}\right)\right. \\
& \left.+\left(\left(\hat{t}-m_{j}^{2}\right)^{2}+m_{j}^{2} \hat{s}\right)\left(\left|\mathcal{D}_{i j L R}\right|^{2}+\left|\mathcal{D}_{i j R L}\right|^{2}\right)\right\}, \quad(30)
\end{aligned}
$$

where

$$
\begin{aligned}
\mathcal{D}_{i j \lambda \lambda^{\prime}}(\hat{s})= & Q_{i} Q_{j} \Delta_{\gamma \gamma}(\hat{s})+\frac{4}{\sin ^{2} 2 \theta_{W}} \zeta_{i \lambda} \zeta_{j \lambda^{\prime}} \Delta_{Z Z}(\hat{s}) \\
& +\frac{2}{\sin 2 \theta_{W}}\left(\zeta_{i \lambda} Q_{j} \Delta_{Z \gamma}(\hat{s})+Q_{i} \zeta_{j \lambda^{\prime}} \Delta_{\gamma Z}(\hat{s})\right) .
\end{aligned}
$$

Finally, the rate for the subprocess $u_{i} \bar{d}_{i} \rightarrow f_{j} \bar{f}_{j}^{\prime}$ is

$$
\frac{d \hat{\sigma}\left(u_{i} \bar{d}_{i} \rightarrow W^{+} \rightarrow f_{j} \bar{f}_{j}^{\prime}\right)}{d \hat{t}}=\frac{N_{f} \pi \alpha^{2}}{12 \sin ^{4} \theta_{W} \hat{s}^{2}}\left(\hat{u}-m_{j}^{2}\right)\left(\hat{u}-m_{j}^{\prime 2}\right)\left|\Delta_{W W}(\hat{s})\right|^{2} .
$$




\section{The Color-Nonsinglet Sector of the TCSM}

The elementary particles of our TC2 model are the $S U(3)_{1} \otimes S U(3)_{2}$ gauge bosons $V_{1}^{A}$ and $V_{2}^{A}(A=1, \ldots, 8)$ and the technifermion doublets $T_{1}=$ $\left(U_{1}, D_{1}\right) \in\left(\mathbf{3}, \mathbf{1}, \mathbf{N}_{\mathbf{T C}}\right)$ and $T_{2}=\left(U_{2}, D_{2}\right) \in\left(\mathbf{1}, \mathbf{3}, \mathbf{N}_{\mathbf{T C}}\right)$ of $S U(3)_{1} \otimes S U(3)_{2} \otimes$ $S U\left(N_{T C}\right)$. The gauge boson mass eigenstates are the $S U(3)_{C}$ gluons $g^{A}$ and the coloron octet $V_{8}^{A}$ :

$$
\begin{aligned}
g^{A} & =\frac{g_{2} V_{1}^{A}+g_{1} V_{2}^{A}}{\sqrt{g_{1}^{2}+g_{2}^{2}}} \equiv \sin \theta_{3} V_{1}^{A}+\cos \theta_{3} V_{2}^{A}, \\
V_{8}^{A} & =\frac{g_{1} V_{1}^{A}-g_{2} V_{2}^{A}}{\sqrt{g_{1}^{2}+g_{2}^{2}}} \equiv \cos \theta_{3} V_{1}^{A}-\sin \theta_{3} V_{2}^{A},
\end{aligned}
$$

where $\alpha_{1}=g_{1}^{2} / 4 \pi \gg \alpha_{2} \simeq \alpha_{C}=g_{C}^{2} / 4 \pi$ and $g_{C}=g_{1} g_{2} / \sqrt{g_{1}^{2}+g_{2}^{2}}$. We shall assume that, near $1 \mathrm{TeV}, S U(3)_{1}$ interactions are nearly strong enough to cause top quark condensation. In the Nambu-Jona-Lasinio approximation, this means $\alpha_{V_{8}} \equiv 4 \alpha_{C} / \sin ^{2} 2 \theta_{3} \gtrsim \pi / 2 C_{2}(\mathbf{3}) \cos ^{4} \theta_{3}$ where $C_{2}(\mathbf{3})=4 / 3$ (see Ref. [29]). With $\alpha_{C} \simeq 0.1$, this requires $\tan \theta_{3} \lesssim \sqrt{0.08}$. In terms of the $S U(3)_{1}$ and $S U(3)_{2}$ currents $j_{1,2 \mu}^{A}$, the fermion-gluon-coloron interactions are

$$
g_{1} j_{1 \mu}^{A} V_{1}^{A \mu}+g_{2} j_{2 \mu}^{A} V_{2}^{A \mu}=g_{C}\left(\cot \theta_{3} j_{1 \mu}^{A}-\tan \theta_{3} j_{2 \mu}^{A}\right) V_{8}^{A \mu}+g_{C} j_{c \mu}^{A} g^{A \mu},
$$

where $j_{c \mu}^{A}=j_{1 \mu}^{A}+j_{2 \mu}^{A}$ is the $S U(3)_{C}$ current.

We consider the couplings of both standard [13] and flavor-universal [28] TC2 models. In standard TC2, top and bottom quarks couple to $S U(3)_{1}$ and the four light quarks to $S U(3)_{2}$. The fermion parts of the $S U(3)_{1,2}$ currents are

$$
\begin{aligned}
& j_{1 \mu}^{A}=\frac{1}{2} \bar{T}_{1} \gamma_{\mu} \lambda_{A} T_{1}+\sum_{a=t, b} \frac{1}{2} \bar{q}_{a} \gamma_{\mu} \lambda_{A} q_{a} \\
& j_{2 \mu}^{A}=\frac{1}{2} \bar{T}_{2} \gamma_{\mu} \lambda_{A} T_{2}+\sum_{a=u, d, c, s} \frac{1}{2} \bar{q}_{a} \gamma_{\mu} \lambda_{A} q_{a} .
\end{aligned}
$$

In this case, colorons decay strongly to top and bottom quarks and weakly to the light quarks. In flavor-universal TC2 all quarks couple to $S U(3)_{1}$, not $S U(3)_{2}$, so that colorons couple to all flavors with equal strength. 
We assume that $S U\left(N_{T C}\right)$, not topcolor $S U(3)_{1} \otimes U(1)_{1}$, interactions dominate the formation of color-nonsinglet $\rho_{T}$ and $\pi_{T}$ states. This is an assumption of convenience; it may be inconsistent with the requirement that $U(1)_{1}$ is instrumental in driving the nondiagonal form of the condensates in Eq. (4). On firmer ground, we assume that techni-isospin is not badly broken by ETC interactions so that the $\rho_{T 8}$ are isosinglets. Then, a useful initial basis for the ground state $\rho_{T 8}$ 's that mix with $g$ and $V_{8}$ is:

$$
\begin{aligned}
& \left|\rho_{11}^{A}\right\rangle=\left(\lambda_{A} / 2\right)_{\alpha \beta}\left|U_{1 \alpha} \bar{U}_{1 \beta}+D_{1 \alpha} \bar{D}_{1 \beta}\right\rangle_{1^{-}} \\
& \left|\rho_{22}^{A}\right\rangle=\left(\lambda_{A} / 2\right)_{\alpha \beta}\left|U_{2 \alpha} \bar{U}_{2 \beta}+D_{2 \alpha} \bar{D}_{2 \beta}\right\rangle_{1^{-}} \\
& \left|\rho_{12}^{A}\right\rangle=\left(\lambda_{A} / 2 \sqrt{2}\right)_{\alpha \beta}\left|U_{1 \alpha} \bar{U}_{2 \beta}+D_{1 \alpha} \bar{D}_{2 \beta}+(1 \leftrightarrow 2)\right\rangle_{1^{-}} \\
& \left|\rho_{12^{\prime}}^{A}\right\rangle=i\left(\lambda_{A} / 2 \sqrt{2}\right)_{\alpha \beta}\left|U_{1 \alpha} \bar{U}_{2 \beta}+D_{1 \alpha} \bar{D}_{2 \beta}-(1 \leftrightarrow 2)\right\rangle_{1^{-}}
\end{aligned}
$$

The first two of these states, $\rho_{11}$ and $\rho_{22}$, mix with $V_{8}$ and $g$. The topcolorbreaking condensate in Eq. $(4),\left\langle\bar{T}_{1 L} T_{2 R}\right\rangle \neq 0$, causes them to mix with $\rho_{12}$ and $\rho_{12}$ also. The $\rho_{T 8}$ mixing with $g$ and $V_{8}$ is purely kinetic and is governed by the $U(2)$ matrices $W_{L, R}^{U, D}$ that diagonalize the $\bar{T}_{L i} T_{R j}$ condensates. ${ }^{7}$ These matrices appear, e.g., in the $S U(3)_{1}$ current as follows: ${ }^{8}$

$$
j_{1 \mu}^{A}=\frac{1}{2} \sum_{T=U, D} \bar{T}_{i L} W_{L i 1}^{T \dagger} \gamma_{\mu} \lambda_{A} W_{L 1 j}^{T} T_{j L}+(L \rightarrow R)+\cdots
$$

Even though technicolor may be the strongest of $T_{1,2}$ 's interactions, the topcolor $S U(3)_{1} \otimes U(1)_{1}$ interactions are not weak perturbations; they must be strong enough near $1 \mathrm{TeV}$ to condense top quarks. Therefore, the $U(1)_{1}$ interactions must be custodial-isospin invariant in the technifermion sector $[33,34]$. On the other hand, the $S U(3)_{2}$ interactions of $T_{2}$ are relatively weak. Thus, the approximate chiral symmetry of $T_{1}$ and $T_{2}$ is

$$
\left[S U(2)_{L} \otimes S U(2)_{R}\right]_{1} \otimes\left[S U(6)_{L} \otimes S U(6)_{R}\right]_{2} .
$$

\footnotetext{
${ }^{7}$ The condensate in Eq. (4) breaks $S U(3)_{1} \otimes S U(3)_{2}$ down to ordinary color, $S U(3)_{C}$. The unitary aligning matrices $W^{U, D}=W_{L}^{U, D}\left(W_{R}^{U, D}\right)^{\dagger}$. See Ref. [32] for a recent discussion of vacuum alignment in technicolor, the physics that gives rise to $W_{L, R}^{U, D}$. It is likely that all elements of these matrices are comparable in magnitude. For economy in the default parameters, we shall take $W_{R}^{U, D}=1$, so that $W^{U, D}=W_{L}^{U, D}$.

${ }^{8}$ In standard TC2, these currents are parity-violating if $W_{L} \neq W_{R}$. In flavor-universal $\mathrm{TC} 2$, the quark currents are purely vectorial because the $W_{L, R}$ are unitary. The same is always true of the $S U(3)_{C}$ currents.
} 
Then technifermion condensation leads to a number of (pseudo)Goldstone boson technipions. Although we assume that the technirhos are too light to decay into a pair of technipions, they may decay into one of them plus a gluon. The relevant technipions are an isosinglet $S U(3)_{C}$ octet $\bar{T}_{2} T_{2}$ state and the color-singlet $\pi_{T}^{0 \prime}$ discussed in Section 1 . The color-octet state is ${ }^{9}$

$$
\left|\pi_{T 8}^{A}\right\rangle=\left(\lambda_{A} / 2\right)_{\alpha \beta}\left|U_{2 \alpha} \bar{U}_{2 \beta}+D_{2 \alpha} \bar{D}_{2 \beta}\right\rangle_{0^{-}} .
$$

In the absence of $\rho_{T 8}$ mixing, only $\rho_{22}$ decays into one of these technipions plus a gluon. The strength of $\rho_{22} \rightarrow g \pi_{T}^{0 \prime}$ is controlled by $\cos \chi^{\prime \prime}$, where $\chi^{\prime \prime}$ is another mixing angle.

For the straw man model, $\pi_{T 8}$ is assumed to decay into either fermionantifermion pairs or two gluons, with the decay rates:

$$
\begin{aligned}
\Gamma\left(\pi_{T 8} \rightarrow \bar{f} f\right) & =\frac{1}{4 \pi F_{T}^{2}} N_{f} p_{f} C_{8 f}^{2} m_{f}^{2} \\
\Gamma\left(\pi_{T 8} \rightarrow g g\right) & =\frac{1}{128 \pi^{3} F_{T}^{2}} \alpha_{C}^{2} C_{8 g}^{2} N_{T C}^{2} M_{\pi_{T 8}}^{3} .
\end{aligned}
$$

Here, $C_{8 f}$ is an ETC-model dependent constant. In Ref. [23] the colored technifermions $T_{1,2}$ do not couple to quarks and leptons. Accordingly, we take the default value $C_{8 f}=0$. To turn on $\pi_{T 8} \rightarrow \bar{b} b$, set $C_{8 b} \simeq 1$. In TC2 models, the $\bar{t} t$ mode has $C_{8 t} \simeq m_{b} / m_{t}$. The number of colors of fermion $f$ is $N_{f}$; its momentum is $p_{f} ; \alpha_{C}$ is the QCD coupling evaluated at the technipion mass; and $C_{8 g}^{2}$ is an $S U(3)_{C}$ Clebsch of order one. We use the color-singlet sector technipion decay constant, $F_{T}=F_{\pi} \sin \chi$. The default values of these and other parameters are tabulated at the end of this paper.

\section{5. $\rho_{T 8}$ and $V_{8}$ Decay Rates}

In low-scale technicolor, color-triplet and octet technipion masses are expected to be a few hundred $\mathrm{GeV}$. As noted, we expect the $\rho_{T 8}$ to decay into

\footnotetext{
${ }^{9}$ This state is essentially the same as the $\eta_{T}$ appearing, e.g., in the one-family technicolor model [24]. However, and this is an important feature of TC2 models, it does not have a strong coupling to $\bar{t} t$ and, therefore, does not appear as a resonance in $\bar{t} t$ production as described in Ref. [35].
} 
$\bar{q} q$ and $g g$ dijets and $g \pi_{T}$. The energy-dependent two-parton decay rates are $\left(\text { for } \rho_{i j} \text { mass } \sqrt{s}\right)^{10}$

$$
\begin{aligned}
\Gamma\left(\rho_{i j} \rightarrow \bar{q}_{a} q_{a}\right) & =\frac{\alpha_{C}}{6}\left|\xi_{g} \delta_{i j}+\frac{g_{a} \xi_{\rho_{i j}} s}{g_{C}\left(s-\mathcal{M}_{V_{8}}^{2}\right)}\right|^{2}\left(1+\frac{2 m_{a}^{2}}{s}\right)\left(s-4 m_{a}^{2}\right)^{\frac{1}{2}} \\
\Gamma\left(\rho_{i j} \rightarrow g g\right) & =\frac{\alpha_{C}^{2} \sqrt{s} \delta_{i j}}{4 \alpha_{\rho_{T}}}
\end{aligned}
$$

where $\mathcal{M}_{V_{8}}^{2}=M_{V_{8}}^{2}-i \sqrt{s} \Gamma_{V_{8}}(s)$, and $\Gamma_{V_{8}}(s)$ is defined in Eq. (45) below. The couplings in Eq. (41) are obtained from Eqs. $(34-37)$; for $W_{R i j}^{U, D}=\delta_{i j}$, they are:

$$
\begin{aligned}
g_{a} & =\left(\begin{array}{l}
g_{C} \cot \theta_{3} \text { for } q_{a}=t, b \\
-g_{C} \tan \theta_{3} \text { for } q_{a}=u, d, c, s
\end{array}\right) \quad \text { (ftandard TC2) } \\
g_{a} & =g_{C} \cot \theta_{3} \\
\alpha_{\rho_{T}} & =\frac{g_{\rho_{T}}^{2}}{4 \pi}=2.91\left(\frac{3}{N_{T C}}\right) \\
\xi_{g} & =\frac{g_{C}}{g_{\rho_{T}}} \\
\xi_{\rho_{11}} & =\frac{2 g_{C}}{g_{\rho_{T}} \sin 2 \theta_{3}}\left[\frac{1}{4}\left(\left|W_{L 11}^{U}\right|^{2}+\left|W_{L 11}^{D}\right|^{2}+2\right)-\sin ^{2} \theta_{3}\right] \\
\xi_{\rho_{22}} & =\frac{2 g_{C}}{g_{\rho_{T}} \sin 2 \theta_{3}}\left[\frac{1}{4}\left(\left|W_{L 12}^{U}\right|^{2}+\left|W_{L 12}^{D}\right|^{2}\right)-\sin ^{2} \theta_{3}\right] \\
\xi_{\rho_{12}} & =\frac{g_{C}}{\sqrt{2} g_{\rho_{T}} \sin 2 \theta_{3}} \operatorname{Re}\left[W_{L 11}^{U *} W_{L 12}^{U}+W_{L 11}^{D *} W_{L 12}^{D}\right] \\
\xi_{\rho_{12^{\prime}}} & =\frac{g_{C}}{\sqrt{2} g_{\rho_{T}} \sin 2 \theta_{3}} \operatorname{Im}\left[W_{L 11}^{U *} W_{L 12}^{U}+W_{L 11}^{D *} W_{L 12}^{D}\right] .
\end{aligned}
$$

It is clear from these expressions how to generalize to $W_{R}^{U, D} \neq 1$. We have assumed that the $\rho_{T 8} \rightarrow \pi_{T} \pi_{T}$ coupling $g_{\rho_{T}}$ is the same for all ground-state

\footnotetext{
${ }^{10}$ Zerwekh and Rosenfeld have argued that hidden local symmetry implies $\rho_{T 8} \rightarrow g g$ is forbidden [36]. However, as Chivukula, Grant and Simmons have shown, hidden local symmetry is inappropriate for bound state $\rho_{T 8}$ 's. The latter authors do emphasize that there is some uncertainty in the strength of the $\rho_{T 8} \rightarrow g g$ amplitude [37]. Nevertheless, we use the canonical decay rate from Ref. [24].
} 
technirhos, and the same that we have used for color singlets; as usual, we use the naive large $-N_{T C}$ value. Using Eq. (5), the $\xi_{\rho_{T 8}}$ parameters become

$$
\begin{aligned}
\xi_{\rho_{11}} & =\frac{2 g_{C}}{g_{\rho_{T}} \sin 2 \theta_{3}}\left[\frac{1}{4}\left(2+\left(W_{L 11}^{U}\right)^{2}+\left(W_{L 11}^{D}\right)^{2}\right)-\sin ^{2} \theta_{3}\right] \\
\xi_{\rho_{22}} & =\frac{2 g_{C}}{g_{\rho_{T}} \sin 2 \theta_{3}}\left[\frac{1}{4}\left(2-\left(W_{L 11}^{U}\right)^{2}-\left(W_{L 11}^{D}\right)^{2}\right)-\sin ^{2} \theta_{3}\right] \\
\xi_{\rho_{12}} & =\frac{g_{C}}{\sqrt{2} g_{\rho_{T}} \sin 2 \theta_{3}}\left[W_{L 11}^{U} \sqrt{1-\left(W_{L 11}^{U}\right)^{2}} \cos \phi_{U}+W_{L 11}^{D} \sqrt{1-\left(W_{L 11}^{D}\right)^{2}} \cos \phi_{D}\right] \\
\xi_{\rho_{12^{\prime}}} & =\frac{g_{C}}{\sqrt{2} g_{\rho_{T}} \sin 2 \theta_{3}}\left[W_{L 11}^{U} \sqrt{1-\left(W_{L 11}^{U}\right)^{2}} \sin \phi_{U}+W_{L 11}^{D} \sqrt{1-\left(W_{L 11}^{D}\right)^{2}} \sin \phi_{D}\right] .
\end{aligned}
$$

Only the $\rho_{22}$ can decay to $g \pi_{T}$; the rates are

$$
\begin{aligned}
\Gamma\left(\rho_{22} \rightarrow g \pi_{T 8}\right) & =\frac{5 \alpha_{C} p^{3}}{9 M_{8}^{2}} \\
\Gamma\left(\rho_{22} \rightarrow g \pi_{T}^{0 \prime}\right) & =\frac{2 \alpha_{C} \cos ^{2} \chi^{\prime \prime} p^{3}}{9 M_{8}^{2}} .
\end{aligned}
$$

Here, $p=\left(s-M_{\pi_{T}}^{2}\right) / 2 \sqrt{s}$ is the gluon or $\pi_{T}$ momentum and $M_{8}$ is a mass parameter of order several hundred $\mathrm{GeV}$, analogous to the parameter $M_{V}$ in Eq. (10). So long as $\pi_{T 8} \rightarrow g g$ is the octet technipion's principal decay mode, it will be difficult, if not impossible, to detect it in $\rho_{22}$ decays.

The $V_{8}$ colorons are expected to be considerably heavier than the $\rho_{T 8}[29$, 31]. Since they couple to quarks, they can decay to dijets; see Eqs $(34,35)$. In both the standard and flavor-universal models, colorons couple strongly to $\bar{T}_{1} T_{1}$, but with strength $g_{C}$ to $\bar{T}_{2} T_{2}$. Since relatively light technipions are $\bar{T}_{2} T_{2}$ states, we expect $\Gamma\left(V_{8} \rightarrow \pi_{T} \pi_{T}\right)=\mathcal{O}\left(\alpha_{C}\right)$ and $\Gamma\left(V_{8} \rightarrow g \pi_{T}\right)=\mathcal{O}\left(\alpha_{C}^{2}\right)$. In this paper, we make the simplifying assumption that such decays are irrelevant. ${ }^{11}$ Then the $V_{8}$ decay rate is the sum over open channels of

$$
\Gamma\left(V_{8} \rightarrow \bar{q}_{a} q_{a}\right)=\frac{\alpha_{a}}{6}\left(1+\frac{2 m_{a}^{2}}{s}\right)\left(s-4 m_{a}^{2}\right)^{\frac{1}{2}},
$$

where $\alpha_{a}=g_{a}^{2} / 4 \pi$ was given in Eq. (42) for the two types of TC2 models we consider.

\footnotetext{
${ }^{11} \mathrm{~A}$ heavy $V_{8}$ may be able to decay to technihadron pairs containing $\bar{T}_{1} T_{1}$. We shall not attempt to include these modes in the $V_{8}$ 's energy-dependent width. This approximation is good at Tevatron energies.
} 


\section{Subprocess Cross Sections for Dijets}

The subprocess cross sections presented below assume massless initial-state partons. They are averaged over initial spins and colors. These cross sections require the propagator matrix describing mixing between the gluon, $V_{8}$ and the $\rho_{T 8}$ 's. We have assumed that the mixing between gauge bosons and $\rho_{T 8}$ is purely kinetic, proportional to $s$. There are also mixing terms $M_{i j, k l}^{2}=M_{k l, i j}^{2}$ between different $\rho_{T 8}$, induced by $\bar{T}_{1} T_{2}$ condensation. They are given by

$$
\begin{aligned}
M_{11,22}^{2} & =\frac{1}{2}\left[\left|W_{12}^{U}\right|^{2}+\left|W_{12}^{D}\right|^{2}\right] M_{8}^{\prime 2}=\frac{1}{2}\left[2-\left(W_{L 11}^{U}\right)^{2}-\left(W_{L 11}^{D}\right)^{2}\right] M_{8}^{\prime 2} \\
M_{11,12}^{2} & =\frac{1}{\sqrt{2}} \operatorname{Re}\left[W_{11}^{U} W_{12}^{U}+W_{11}^{D} W_{12}^{D}\right] M_{8}^{\prime 2} \\
& =\frac{1}{\sqrt{2}}\left[W_{L 11}^{U} \sqrt{1-\left(W_{L 11}^{U}\right)^{2}} \cos \phi_{U}+W_{L 11}^{D} \sqrt{1-\left(W_{L 11}^{D}\right)^{2}} \cos \phi_{D}\right] M_{8}^{\prime 2} \\
M_{11,12^{\prime}}^{2} & =-\frac{1}{\sqrt{2}} \operatorname{Im}\left[W_{11}^{U} W_{12}^{U}+W_{11}^{D} W_{12}^{D}\right] M_{8}^{\prime 2} \\
& =-\frac{1}{\sqrt{2}}\left[W_{L 11}^{U} \sqrt{1-\left(W_{L 11}^{U}\right)^{2}} \sin \phi_{U}+W_{L 11}^{D} \sqrt{1-\left(W_{L 11}^{D}\right)^{2}} \sin \phi_{D}\right] M_{8}^{\prime 2} \\
M_{22,12}^{2} & =\frac{1}{\sqrt{2}} \operatorname{Re}\left[W_{21}^{U} W_{22}^{U}+W_{21}^{D} W_{22}^{D}\right] M_{8}^{\prime 2} \\
& =-\frac{1}{\sqrt{2}}\left[W_{L 11}^{U} \sqrt{1-\left(W_{L 11}^{U}\right)^{2}} \cos \phi_{U}+W_{L 11}^{D} \sqrt{1-\left(W_{L 11}^{D}\right)^{2}} \cos \phi_{D}\right] M_{8}^{\prime 2} \\
M_{22,12^{\prime}}^{2} & =\frac{1}{\sqrt{2}} \operatorname{Im}\left[W_{21}^{U} W_{22}^{U}+W_{21}^{D} W_{22}^{D}\right] M_{8}^{\prime 2} \\
& =\frac{1}{\sqrt{2}}\left[W_{L 11}^{U} \sqrt{1-\left(W_{L 11}^{U}\right)^{2}} \sin \phi_{U}+W_{L 11}^{D} \sqrt{1-\left(W_{L 11}^{D}\right)^{2}} \sin \phi_{D}\right] M_{8}^{\prime 2} \\
& =-\frac{1}{2} \operatorname{Im}\left[\left(W_{12}^{U}\right)^{2}+\left(W_{12}^{D}\right)^{2}\right] M_{8}^{\prime 2} \\
M_{12,12^{\prime}}^{2} & =\frac{1}{2}\left[\left(1-\left(W_{L 11}^{U}\right)^{2}\right) \sin _{2} \phi_{U}+\left(1-\left(W_{L 11}^{D}\right)^{2}\right) \sin 2 \phi_{D}\right] M_{8}^{\prime 2} . \\
& =-14
\end{aligned}
$$

Here, $M_{8}^{\prime}$ is another technicolor scale mass parameter of order $M_{8}$. In the second equalities, we assumed $W_{R}^{U, D}=1$ and used Eq. (5)). The $g-V_{8}-\rho_{11}-$ 
$\rho_{22}-\rho_{12}-\rho_{12^{\prime}}$ propagator is the inverse of the symmetric matrix

$$
D^{-1}(s)=\left(\begin{array}{cccccc}
s & 0 & s \xi_{g} & s \xi_{g} & 0 & 0 \\
0 & s-\mathcal{M}_{V_{8}}^{2} & s \xi_{\rho_{11}} & s \xi_{\rho_{22}} & s \xi_{\rho_{12}} & s \xi_{\rho_{12^{\prime}}} \\
s \xi_{g} & s \xi_{\rho_{11}} & s-\mathcal{M}_{11}^{2} & -M_{11,22}^{2} & -M_{11,12}^{2} & -M_{11,12^{\prime}}^{2} \\
s \xi_{g} & s \xi_{\rho_{22}} & -M_{11,22}^{2} & s-\mathcal{M}_{22}^{2} & -M_{22,12}^{2} & -M_{22,12^{\prime}}^{2} \\
0 & s \xi_{\rho_{12}} & -M_{11,12}^{2} & -M_{22,12}^{2} & s-\mathcal{M}_{12}^{2} & -M_{12,12^{\prime}}^{2} \\
0 & s \xi_{\rho_{12^{\prime}}} & -M_{11,12^{\prime}}^{2} & -M_{22,12^{\prime}}^{2} & -M_{12,12^{\prime}}^{2} & s-\mathcal{M}_{12^{\prime}}^{2}
\end{array}\right) .
$$

Here, $\mathcal{M}_{V}^{2}=M_{V}^{2}-i \sqrt{s} \Gamma_{V}(s)$ uses the energy-dependent widths of the octet vector bosons. The following combinations of propagators are used in the parton-parton cross sections (here, $q_{a, b}=u, d, c, s$ or $\left.t, b\right)$ :

$$
\begin{aligned}
\mathcal{D}_{q_{a} q_{b}}(s) & =D_{g g}(s)+\left(\frac{g_{a}+g_{b}}{g_{C}}\right) D_{g V_{8}}(s)+\frac{g_{a} g_{b}}{g_{C}^{2}} D_{V_{8} V_{8}}(s) \\
\Delta_{g g}(s) & =s D_{g g}(s)-1 \\
\mathcal{D}_{q_{a} g}(s) & =D_{g g}(s)+\frac{g_{a}}{g_{C}} D_{g V_{8}}(s) \\
\Delta_{q_{a} g}(s) & =s \mathcal{D}_{q_{a} g}(s)-1 .
\end{aligned}
$$

The subprocess cross sections for $2 \rightarrow 2$ scattering of light quarks $u, d, c, s, b$ and gluons are given by (here $q_{a} \neq q_{b}$ ):

$$
\begin{aligned}
\frac{d \hat{\sigma}\left(\bar{q}_{a} q_{a} \rightarrow \bar{q}_{b} q_{b}\right)}{d \hat{t}} & =\frac{4 \pi \alpha_{C}^{2}}{9 \hat{s}^{2}}\left|\mathcal{D}_{q_{a} q_{b}}(\hat{s})\right|^{2}\left(\hat{u}^{2}+\hat{t}^{2}\right) \\
\frac{d \hat{\sigma}\left(q_{a} q_{b} \rightarrow q_{a} q_{b}\right)}{d \hat{t}} & =\frac{d \hat{\sigma}\left(\bar{q}_{a} \bar{q}_{b} \rightarrow \bar{q}_{a} \bar{q}_{b}\right)}{d \hat{t}}=\frac{4 \pi \alpha_{C}^{2}}{9 \hat{s}^{2}}\left|\mathcal{D}_{q_{a} q_{b}}(\hat{t})\right|^{2}\left(\hat{u}^{2}+\hat{s}^{2}\right) \\
\frac{d \hat{\sigma}\left(\bar{q}_{a} q_{a} \rightarrow \bar{q}_{a} q_{a}\right)}{d \hat{t}} & =\frac{4 \pi \alpha_{C}^{2}}{9 \hat{s}^{2}}\left\{\left|\mathcal{D}_{q_{a} q_{a}}(\hat{s})\right|^{2}\left(\hat{u}^{2}+\hat{t}^{2}\right)+\left|\mathcal{D}_{q_{a} q_{a}}(\hat{t})\right|^{2}\left(\hat{u}^{2}+\hat{s}^{2}\right)\right. \\
\frac{d \hat{\sigma}\left(q_{a} q_{a} \rightarrow q_{a} q_{a}\right)}{d \hat{t}} & =\frac{\left.-\frac{2}{3} \operatorname{Re}\left(\mathcal{D}_{q_{a} q_{a}}(\hat{s}) \mathcal{D}_{q_{a} q_{a}}^{*}(\hat{t})\right) \hat{u}^{2}\right\}}{d \hat{\sigma}\left(\bar{q}_{a} \bar{q}_{a} \rightarrow \bar{q}_{a} \bar{q}_{a}\right)}
\end{aligned}
$$




$$
\begin{aligned}
& =\frac{2 \pi \alpha_{C}^{2}}{9 \hat{s}^{2}}\left\{\left|\mathcal{D}_{q_{a} q_{a}}(\hat{t})\right|^{2}\left(\hat{u}^{2}+\hat{s}^{2}\right)+\left|\mathcal{D}_{q_{a} q_{a}}(\hat{u})\right|^{2}\left(\hat{t}^{2}+\hat{s}^{2}\right)\right. \\
& \left.-\frac{2}{3} \operatorname{Re}\left(\mathcal{D}_{q_{a} q_{a}}(\hat{t}) \mathcal{D}_{q_{a} q_{a}}^{*}(\hat{u})\right) \hat{s}^{2}\right\} \\
& \frac{d \hat{\sigma}\left(g g \rightarrow \bar{q}_{a} q_{a}\right)}{d \hat{t}}=\frac{9}{32} \frac{d \hat{\sigma}\left(\bar{q}_{a} q_{a} \rightarrow g g\right)}{d \hat{t}} \\
& =\frac{3 \pi \alpha_{C}^{2}}{8 \hat{s}^{2}}\left\{\frac{4}{9}\left(\frac{\hat{u}}{\hat{t}}+\frac{\hat{t}}{\hat{u}}\right)-\left(\frac{\hat{t}^{2}+\hat{u}^{2}}{\hat{s}^{2}}\right)+\frac{2 \hat{t} \hat{u}}{\hat{s}^{2}}\left|\Delta_{q_{a g}}(\hat{s})\right|^{2}\right\} \\
& \frac{d \hat{\sigma}\left(q_{a} g \rightarrow q_{a} g\right)}{d \hat{t}}=\frac{\pi \alpha_{C}^{2}}{\hat{s}^{2}}\left\{\left(\frac{\hat{s}^{2}+\hat{u}^{2}}{\hat{t}^{2}}\right)-\frac{4}{9}\left(\frac{\hat{u}}{\hat{s}}+\frac{\hat{s}}{\hat{u}}\right)-\frac{2 \hat{s} \hat{u}}{\hat{t}^{2}}\left|\Delta_{q_{a} g}(\hat{t})\right|^{2}\right\} \\
& \frac{d \hat{\sigma}(g g \rightarrow g g)}{d \hat{t}}=\frac{9 \pi \alpha_{c}^{2}}{32 \hat{s}^{2}}\left\{\left|\Delta_{g g}(\hat{s})\left(\frac{\hat{t}-\hat{u}}{\hat{s}}\right)+\Delta_{g g}(\hat{t})\left[\frac{\hat{u}^{2}}{\hat{s}^{2}}\left(\frac{\hat{s}-\hat{u}}{\hat{t}}\right)+\frac{8 \hat{u}}{\hat{s}}\right]+\frac{2 \hat{s}}{\hat{t}}\right|^{2}\right. \\
& +\left|\Delta_{g g}(\hat{s})\left(\frac{\hat{u}-\hat{t}}{\hat{s}}\right)+\Delta_{g g}(\hat{u})\left[\frac{\hat{t}^{2}}{\hat{s}^{2}}\left(\frac{\hat{s}-\hat{t}}{\hat{u}}\right)+\frac{8 \hat{t}}{\hat{s}}\right]+\frac{2 \hat{s}}{\hat{u}}\right|^{2} \\
& +\operatorname{Re}\left[\left(\Delta_{g g}^{*}(\hat{s})\left(\frac{\hat{t}-\hat{u}}{\hat{s}}\right)+\Delta_{g g}^{*}(\hat{t})\left[\frac{\hat{u}^{2}}{\hat{s}^{2}}\left(\frac{\hat{s}-\hat{u}}{\hat{t}}\right)+\frac{8 \hat{u}}{\hat{s}}\right]+\frac{2 \hat{s}}{\hat{t}}\right)\right. \\
& \left.\times\left(\Delta_{g g}(\hat{s})\left(\frac{\hat{u}-\hat{t}}{\hat{s}}\right)+\Delta_{g g}(\hat{u})\left[\frac{\hat{t}^{2}}{\hat{s}^{2}}\left(\frac{\hat{s}-\hat{t}}{\hat{u}}\right)+\frac{8 \hat{t}}{\hat{s}}\right]+\frac{2 \hat{s}}{\hat{u}}\right)\right] \\
& +\left|\Delta_{g g}(\hat{s})\left(\frac{\hat{t}-\hat{u}}{\hat{s}}\right)+\Delta_{g g}(\hat{t})\left(\frac{\hat{u}^{2}-\hat{s}^{2}}{\hat{s}^{2}}\right)\right|^{2} \\
& +\left|\Delta_{g g}(\hat{s})\left(\frac{\hat{u}-\hat{t}}{\hat{s}}\right)+\Delta_{g g}(\hat{u})\left(\frac{\hat{t}^{2}-\hat{s}^{2}}{\hat{s}^{2}}\right)\right|^{2} \\
& +\operatorname{Re}\left[\left(\Delta_{g g}^{*}(\hat{s})\left(\frac{\hat{t}-\hat{u}}{\hat{s}}\right)+\Delta_{g g}^{*}(\hat{t})\left(\frac{\hat{u}^{2}-\hat{s}^{2}}{\hat{s}^{2}}\right)\right)\right. \\
& \left.\times\left(\Delta_{g g}(\hat{s})\left(\frac{\hat{u}-\hat{t}}{\hat{s}}\right)+\Delta_{g g}(\hat{u})\left(\frac{\hat{t}^{2}-\hat{s}^{2}}{\hat{s}^{2}}\right)\right)\right] \\
& +\left(\frac{\hat{u}^{4}+\hat{t}^{4}}{\hat{s}^{4}}\right)\left[\left|\Delta_{g g}(\hat{t})\left(\frac{\hat{s}-\hat{u}}{\hat{t}}\right)+\frac{2 \hat{s}}{\hat{t}}\right|^{2}+\left|\Delta_{g g}(\hat{u})\left(\frac{\hat{s}-\hat{t}}{\hat{u}}\right)+\frac{2 \hat{s}}{\hat{u}}\right|^{2}\right. \\
& \left.+\operatorname{Re}\left[\left(\Delta_{g g}^{*}(\hat{t})\left(\frac{\hat{s}-\hat{u}}{\hat{t}}\right)+\frac{2 \hat{s}}{\hat{t}}\right)\left(\Delta_{g g}(\hat{u})\left(\frac{\hat{s}-\hat{t}}{\hat{u}}\right)+\frac{2 \hat{s}}{\hat{u}}\right)\right]\right]
\end{aligned}
$$




$$
\left.+4\left(\frac{\hat{t} \hat{u}}{\hat{s}^{2}}\right)^{2}\left[\left|\Delta_{g g}(\hat{t})\right|^{2}+\left|\Delta_{g g}(\hat{u})\right|^{2}+\operatorname{Re}\left(\Delta_{g g}^{*}(\hat{t}) \Delta_{g g}(\hat{u})\right)\right]\right\} .
$$

For massless partons, $\hat{t}=-\frac{1}{2} \hat{s}(1-\cos \theta)$ and $\hat{u}=-\frac{1}{2} \hat{s}(1+\cos \theta)$ where $\theta$ is the cm scattering angle.

The cross sections for top quark production are:

$$
\begin{aligned}
\frac{d \hat{\sigma}\left(\bar{q}_{a} q_{a} \rightarrow \bar{t} t\right)}{d \hat{t}}=\frac{4 \pi \alpha_{C}^{2}}{9 \hat{s}^{4}}\left|\hat{s} \mathcal{D}_{q_{a}}(\hat{s})\right|^{2}\left[\left(\hat{u}-m_{t}^{2}\right)^{2}+\left(\hat{t}-m_{t}^{2}\right)^{2}+2 m_{t}^{2} \hat{s}\right] \\
\frac{d \hat{\sigma}(g g \rightarrow \bar{t} t)}{d \hat{t}}=\frac{3 \pi \alpha_{C}^{2}}{8 \hat{s}^{2}}\left\{\frac { 4 } { 9 } \left[\frac{\left(\hat{t}-m_{t}^{2}\right)\left(\hat{u}-m_{t}^{2}\right)-2 m_{t}^{2}\left(\hat{t}+m_{t}^{2}\right)}{\left(\hat{t}-m_{t}^{2}\right)^{2}}\right.\right. \\
\left.\quad+\frac{\left(\hat{t}-m_{t}^{2}\right)\left(\hat{u}-m_{t}^{2}\right)-2 m_{t}^{2}\left(\hat{u}+m_{t}^{2}\right)}{\left(\hat{u}-m_{t}^{2}\right)^{2}}\right] \\
+\frac{\left(\hat{t}-m_{t}^{2}\right)\left(\hat{u}-m_{t}^{2}\right)+m_{t}^{2}(\hat{t}-\hat{u}) \operatorname{Re}\left(\Delta_{t g}(\hat{s})-1\right)}{\hat{s}\left(\hat{t}-m_{t}^{2}\right)} \\
+\frac{\left(\hat{t}-m_{t}^{2}\right)\left(\hat{u}-m_{t}^{2}\right)+m_{t}^{2}(\hat{u}-\hat{t}) \operatorname{Re}\left(\Delta_{t g}(\hat{s})-1\right)}{\hat{s}\left(\hat{u}-m_{t}^{2}\right)} \\
\left.+\frac{2}{\hat{s}^{2}}\left(\hat{t}-m_{t}^{2}\right)\left(\hat{u}-m_{t}^{2}\right)\left[\left|\Delta_{t g}(\hat{s})\right|^{2}+1\right]-\frac{m_{t}^{2}\left(\hat{s}-4 m_{t}^{2}\right)}{9\left(\hat{t}-m_{t}^{2}\right)\left(\hat{u}-m_{t}^{2}\right)}\right\} .
\end{aligned}
$$

Here, $\hat{t}=m_{t}^{2}-\frac{1}{2} \hat{s}\left(1-\beta_{t} \cos \theta\right)$ where $\beta_{t}=\sqrt{1-4 m_{t}^{2} / \hat{s}}$.

\section{Hadron Collider Phenomenology}

In this section, we concentrate on the phenomenology of the color-octet sector of the TCSM. The color-singlet sector has been studied in Refs. [1, $3,4,5,6,7,16,17,21]$. Numerical results are presented for the Tevatron collider, since new strong interactions may be visible there if they are related to electroweak symmetry breaking and the top quark's mass. Our results are readily generalizable to the $\mathrm{LHC}$.

The impact of the coloron $V_{8}$ on high- $p_{T}$ scattering has been explored in the past [31, 38, 39] and, by itself, it is easy to understand. The improvement in the present approach is to include the full propagator structure, with an 
energy-dependent width $\Gamma_{V_{8}}(s)$ for the coloron. The simplification of treating coloron-exchange as a contact term is appropriate when the coloron is very heavy and $\sqrt{s} \Gamma_{V_{8}}(s) \ll M_{V_{8}}^{2}$.

Let us first ignore the subleading effects of kinetic mixing with the coloroctet technirhos. Then, in fermion scattering processes, the coloron appears as a modification to the gluon propagator which may depend on the fermion generations. For standard TC2, there is an enhanced coloron coupling to $b$ and $t$ quarks, which changes the gluon propagator connecting pairs of fermions to

$$
\mathcal{D}_{q t}(s)=\frac{1}{s}\left[1-\frac{s}{s-M_{V_{8}}^{2}-i \sqrt{s} \Gamma_{V_{8}}(s)}\right] .
$$

In the limit $M_{V_{8}} \gg m_{t}$, the energy dependent width scales as $\sqrt{s}$. For the "NJL-inspired" value of the topcolor $S U(3)$ mixing angle, $\tan \theta_{3}=\sqrt{.08}$, the coloron width is roughly $\sqrt{s} / 3$. Below resonance, the coloron interferes constructively, and can thus manifest itself as an energy-dependent enhancement of the $b$ or $t$ quark cross sections. The invariant mass distribution of top quark pairs $\mathcal{M}_{\bar{t} t}$ was measured by both $\mathrm{CDF}$ and $\mathrm{D} \varnothing[40,41]$, and it constrains the values of $M_{V_{8}}$ and $\tan \theta_{3}$ (the latter dependence arises indirectly through the coloron width). Roughly, these measurements are consistent with a coloron mass near $1 \mathrm{TeV}$, with little restriction on the value of $\tan \theta_{3}$. ${ }^{12}$ From the $\mathcal{M}_{\bar{b} b}$ distribution, CDF [19] excludes standard TC2 colorons in the mass range $280-670 \mathrm{GeV}$ for $\Gamma_{V_{8}} / M_{V_{8}}=0.3$. The limit worsens as the width is increased, essentially vanishing for $\Gamma_{V_{8}} / M_{V_{8}}>0.7$.

For flavor-universal TC2, there is an enhanced coloron coupling to all flavors of quarks:

$$
\mathcal{D}_{q q^{\prime}}(s)=\frac{1}{s}\left[1+\frac{1}{\tan ^{2} \theta_{3}} \frac{s}{s-M_{V_{8}}^{2}-i \sqrt{s} \Gamma_{V_{8}}(s)}\right] .
$$

In contrast to standard $\mathrm{TC} 2$, the coloron interferes destructively below resonance, almost cancelling the simple gluon exchange at $\sqrt{s}=M_{V_{8}} \sin \theta_{3}$. Furthermore, the on-mass-shell coloron width is comparable to its mass for our default choice of parameters. We shall see below that consistency with the $\mathrm{CDF}$ and $\varnothing \varnothing$ data tends to require a universal coloron mass of several $\mathrm{TeV}$, in

\footnotetext{
${ }^{12}$ This and the following statement for the universal coloron are based on basic fits to the public Run I data, but a more systematic analysis is needed. There are no published results on limits for wide resonances in the $\bar{t} t$ system.
} 
agreement with the $\mathrm{D} \varnothing$ limit resulting from dijet measurements [31]. Despite the fact that the interference occurs for all flavors of quarks in flavor-universal $\mathrm{TC} 2$, the $\mathcal{M}_{\bar{t} t}$ distribution itself ultimately may be the most constraining, because of the relative importance of $\bar{q} q$ annihilation to other partonic processes. In dijet cross sections, many different subprocesses contribute, some with $s$-channel gluon exchanges (which are destructive below resonance) and some with $t$ and $u$-channel exchanges (which are constructive).

While the coloron phenomenology is straightforward, the effects of the color-octet technirhos are less transparent. To simplify the discussion, we consider first the case of a coloron and a single technirho mixing with the gluon and coloron. The inverse propagator matrix describing the gluon- $V_{8^{-}}$ $\rho_{T 8}$ mixing is

$$
\mathcal{D}_{0}^{-1}(s)=\left(\begin{array}{ccc}
s & 0 & s \xi \\
0 & s-\mathcal{M}_{V_{8}}^{2} & s \xi^{\prime} \\
s \xi & s \xi^{\prime} & s-\mathcal{M}_{\rho}^{2}
\end{array}\right),
$$

where $\xi=\xi_{g}$ and $\xi^{\prime}=\xi_{\rho_{i j}}$ set the strength of the kinetic mixing. The cross sections depend on the propagators (see Eq. 48):

$$
\begin{aligned}
D_{g g} & =\frac{\left(s-\mathcal{M}_{V_{8}}^{2}\right)\left(s-\mathcal{M}_{\rho}^{2}\right)-s^{2} \xi^{2}}{\operatorname{Det} \mathcal{D}_{0}^{-1}}=\frac{1}{s}+\frac{s\left(s-\mathcal{M}_{V_{8}}^{2}\right) \xi^{2}}{\operatorname{Det} \mathcal{D}_{0}^{-1}} \\
D_{g V_{8}} & =\frac{s^{2} \xi \xi^{\prime}}{\operatorname{Det} \mathcal{D}_{0}^{-1}} \\
D_{V_{8} V_{8}} & =\frac{s\left(s-\mathcal{M}_{\rho}^{2}\right)-s^{2} \xi^{2}}{\operatorname{Det} \mathcal{D}_{0}^{-1}}=\frac{1}{\left(s-\mathcal{M}_{V_{8}}^{2}\right)}+\frac{s^{3} \xi^{\prime 2}}{\left(s-\mathcal{M}_{V_{8}}^{2}\right) \operatorname{Det} \mathcal{D}_{0}^{-1}} \\
\operatorname{Det} \mathcal{D}_{0}^{-1} & =s\left(s-\mathcal{M}_{V_{8}}^{2}\right)\left(s-\mathcal{M}_{\rho}^{2}\right)-s^{2}\left(s \xi^{\prime 2}+\left(s-\mathcal{M}_{V_{8}}^{2}\right) \xi^{2}\right)
\end{aligned}
$$

When written in this form, the gluon and coloron components are approximately separated from the technirho's.

The $\bar{t} t$ cross section involves $\mathcal{D}_{q t}$, which depends on the specific model. For standard TC2, it has the form

$$
\begin{gathered}
\mathcal{D}_{q t}=\frac{1}{s}-\frac{1}{s-\mathcal{M}_{V_{8}}^{2}}+ \\
\frac{1}{\operatorname{Det} \mathcal{D}_{0}^{-1}}\left[s\left(s-\mathcal{M}_{V_{8}}^{2}\right) \xi^{2}+s^{2} \xi \xi^{\prime}\left(-\tan \theta_{3}+\frac{1}{\tan \theta_{3}}\right)-\frac{s^{3} \xi^{\prime 2}}{s-\mathcal{M}_{V_{8}}^{2}}\right] .
\end{gathered}
$$


The technirho phenomenology is determined by the last term. Taking $\rho=$ $\rho_{22}$, e.g., this can be written as $\xi^{2} s^{2}$ times the dimensionless quantity

$$
\mathcal{N}=r-\tan ^{2} \theta_{3} / r\left(\frac{1-W^{2}}{2 \sin ^{2} \theta_{3}}-1\right)^{2}+\left(1-\tan ^{2} \theta_{3}\right)\left(\frac{1-W^{2}}{2 \sin ^{2} \theta_{3}}-1\right)
$$

with $r=1-\mathcal{M}_{V_{8}}^{2} / s$ and we put $W=W_{11}^{U}=W_{11}^{D}$.

For small coloron-technirho mixing, $\xi \gg\left|\xi^{\prime}\right|$, the technirho contribution to $\mathcal{D}_{q t}$ reduces to $\xi^{2} /\left(s-\mathcal{M}_{\rho}^{2}\right)$, yielding the behavior of a narrow resonance. For comparable $\xi$ and $\xi^{\prime}$, the maximum of $\mathcal{D}_{q t}$ near $s=M_{\rho}^{2}$ is no longer just inversely proportional to $\Gamma_{\rho}$, but also depends on $\xi^{\prime 2} \Gamma_{V_{8}} / r^{2}$. The effective technirho width is larger and the cross section is smaller, depending on the mixing and coloron properties. Furthermore, certain relations may exist among the parameters in $\mathcal{N}$ that weaken the resonant behavior. For example, the numerator vanishes for $r=\xi^{\prime} / \xi \tan \theta_{3}$ or $r=-\xi^{\prime} / \xi \cot \theta_{3}$. We generally expect that technirhos are lighter than the coloron, so only the latter solution is relevant. This relation is difficult to satisfy unless $W$ is small. As an example, for $W=0, r=(1 / .4)^{2}$, and $\tan \theta_{3}=0.295$, we have $\mathcal{N}=0$. Similar remarks hold in the case of the flavor-universal coloron, where the simplified propagator has the form

$$
\begin{gathered}
\mathcal{D}_{q t}=\frac{1}{s}+\frac{\cot ^{2} \theta_{3}}{s-\mathcal{M}_{V_{8}}^{2}}+ \\
\frac{1}{\operatorname{Det} \mathcal{D}_{0}^{-1}}\left[s\left(s-\mathcal{M}_{V_{8}}^{2}\right) \xi^{2}+2 s^{2} \xi \xi^{\prime} \cot \theta_{3}+\frac{s^{3} \xi^{\prime 2} \cot ^{2} \theta_{3}}{s-\mathcal{M}_{V_{8}}^{2}}\right] .
\end{gathered}
$$

In particular, the same condition, $r=-\xi^{\prime} / \xi \cot \theta_{3}$, makes the numerator factor corresponding to $\mathcal{N}$ vanish.

While this one-technirho example illustrates the propagator structure, the full TC2 model's additional $\rho_{T 8}$ 's and their mixing can lead to substantially more complicated spectra. In addition, the $\rho_{22}$ can have significant $g \pi_{T}^{0 \prime}$ and $g \pi_{T 8}$ decay rates that decrease the cross section on resonance. The resultant phenomenology can be quite rich. Here, we focus on a several benchmark models that demonstrate some interesting features. For further background, we note that CDF placed limits on the additional cross section from narrow resonances $(\Gamma / M<0.1)$ in the $\mathcal{M}_{\bar{b} b}[19]$ and $\mathcal{M}_{\bar{t} t}[40]$ distributions. For invariant masses less than about $550 \mathrm{GeV}, \mathcal{M}_{\bar{b} b}$ is more sensitive, 
while $\mathcal{M}_{\bar{t} t}$ is more sensitive above. Neither of these Run I limits excludes a single, isolated technirho in either the $\bar{b} b$ or $\bar{t} t$ channels. It is not a trivial matter to apply these limits to the cases when several technirhos have comparable mass or when the coloron is relevant.

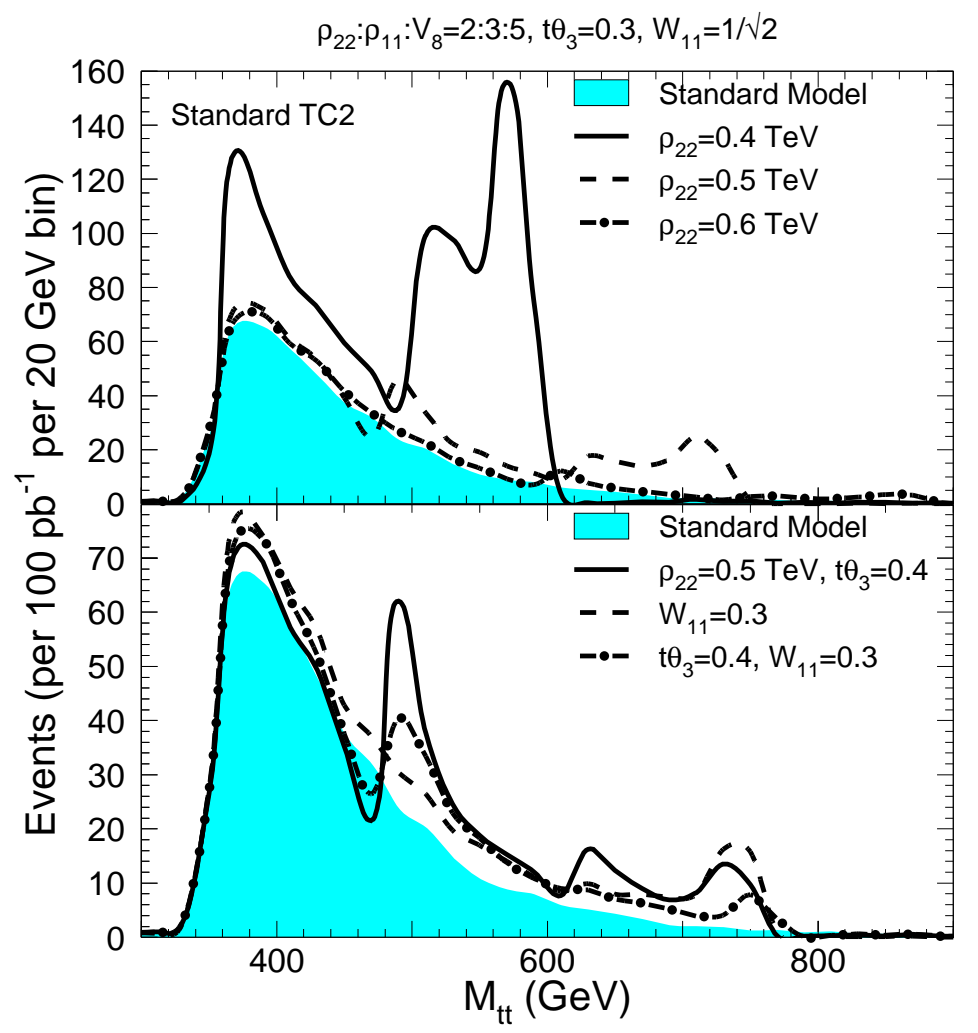

Figure 1: Invariant mass distributions of top quark pairs $\mathcal{M}_{\overline{t t}}$ compared to the standard model distribution for several choices of parameters in standard TC2.

Benchmark I: Standard TC2- $\mathcal{M}_{\bar{t} t}$ with various $M_{\rho_{T 8}}$

This benchmark studies effects from Standard TC2 on the invariant mass distribution of $\bar{t} t$ pairs produced at the Tevatron in Run $\mathrm{I}(\sqrt{s}=1.8 \mathrm{TeV}$ and $\left.\int \mathcal{L} d t=100 \mathrm{pb}^{-1}\right)$. The curves were generated using PүтнIA, but only at the parton level, and with no efficiency or resolution effects. The masses of the $\rho_{22}, \rho_{11}$ and $V_{8}$ were fixed in the ratio 2:3:5, with the $\rho_{12}$ and $\rho_{21}$ degenerate in 
mass at the average of $M_{\rho_{11}}$ and $M_{\rho_{22}} .{ }^{13}$ We set the mass $M_{\pi_{T 8}}=300 \mathrm{GeV}$. Initially, we choose $\tan \theta_{3}=0.3$ and $W=1 / \sqrt{2}$; all other parameters are given by their default values in Table 3. For normalization, the standard model distribution is shown as the filled histogram in Fig. 1. As $M_{\rho_{22}}$ (and hence all other masses from the fixed ratio) is increased from $0.4 \mathrm{TeV}$ (solid line, upper panel) to $0.5 \mathrm{TeV}$ (dashes) to $0.6 \mathrm{TeV}$ (dash-dot), narrow peaks occur with roughly the same relative enhancement over the standard model distribution: the value of $\mathcal{N}=-3.33$ is the same at $\sqrt{s}=M_{\rho_{22}}$ in each case. The absolute size of the peaks decreases with increased mass because of the decreased partonic luminosity. We also studied the dependence of these results on $W$ and $\tan \theta_{3}$ for a fixed value of $\rho_{22}=0.5 \mathrm{TeV}$ (lower panel). First $\tan \theta_{3}$ is increased (solid) with all other parameters at the baseline value, second $W$ is decreased (dashes) with all other parameters at the baseline value, and third both $\tan \theta_{3}$ and $W$ are varied simultaneously (dash-dot) with all other parameters fixed. In these last three cases, the value of $\mathcal{N}$ at $\sqrt{s}=M_{\rho_{22}}$ varies from -4.55 to -0.80 to -3.16 , which is reflected in the relative height of the peaks. The latter should be compared to the baseline value of $\mathcal{N}=-3.33$. Note that none of the individual resonances is excludable with Run I data; a full shape analysis may be sensitive to the first set of parameters (upper panel, solid line). A promising model-line for study by the experiments is to vary $M_{\rho_{22}}$ with the mass ratio fixed and for specific values of $W$ and $\tan \theta_{3}$. It would also be interesting to vary the parameter $M_{8}^{\prime}$, which induces hard mixing between the $\rho_{T 8}$ 's in Eq. (46). As apparent from the plots, the technirho pole masses are shifted from the value of the input mass parameter because of the off-diagonal terms there.

One can also consider the $\bar{b} b$ final state to complement the $\bar{t} t$ distributions. While there are several sources of $\bar{b} b$ production, the largest effect is expected to occur in the direct production mechanism. This can be isolated by requiring two, high- $p_{T}, b$-tagged jets that are balanced azimuthally $(\Delta \phi \sim \pi)$. While such a search would give sensitivity to $\rho_{T 8}$ states below the $\bar{t} t$ threshold, it would not greatly improve the search for higher mass states. Benchmark II: Flavor-Universal TC2- $\mathcal{M}_{\bar{t} t}$ with various $M_{\rho_{T 8}}$

This benchmark studies the impact of flavor-universal TC2. The parameter choices are identical to the case of Benchmark I for standard TC2,

\footnotetext{
${ }^{13}$ This pattern of technirho masses is assumed throughout the rest of this analysis, and is motivated by a valence technifermion approximation.
} 


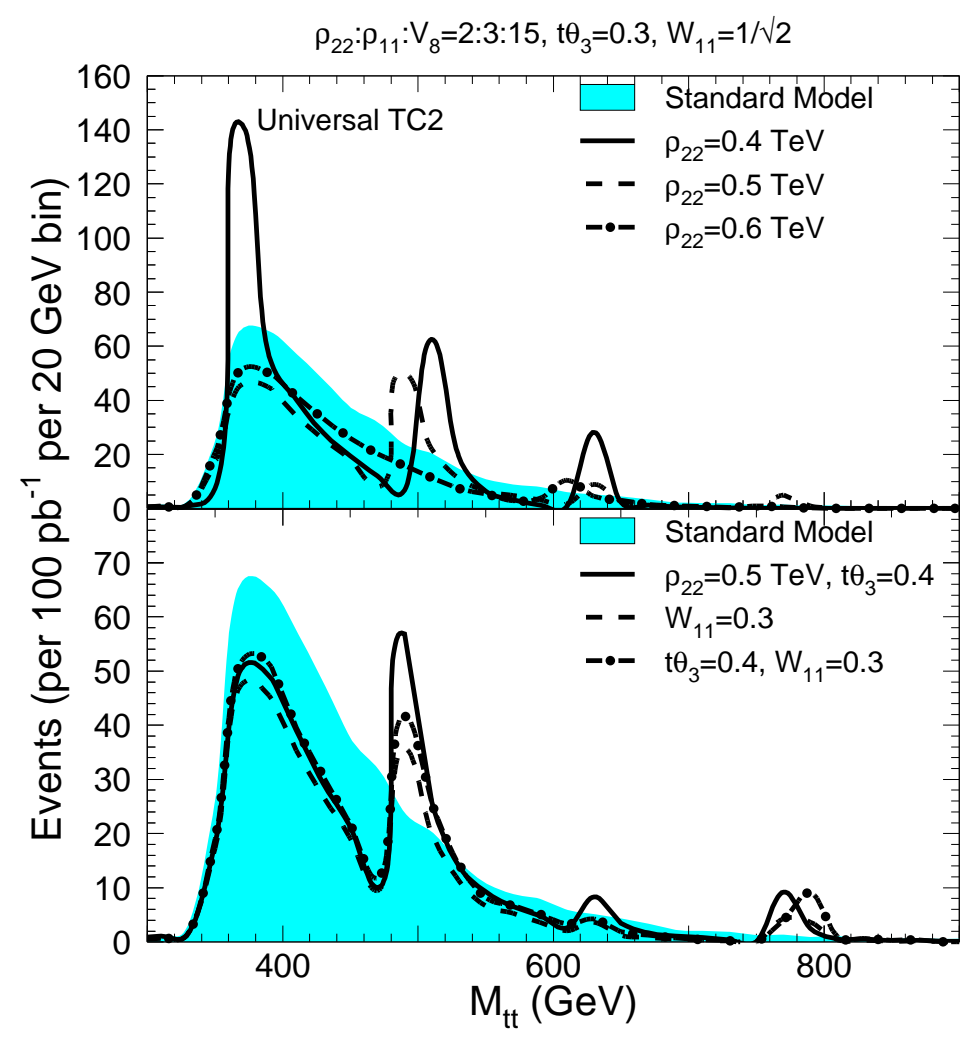

Figure 2: Invariant mass distributions of top quark pairs $\mathcal{M}_{\bar{t} t}$ compared to the standard model distribution for several parameter choices in flavor-universal TC2.

except that the masses of the $\rho_{22}, \rho_{11}$ and $V_{8}$ fixed in the ratio 2:3:15, because of the coloron's stronger coupling in these models. The results are displayed in Fig. 2. The $\mathcal{M}_{\bar{t} t}$ distribution is distorted both by the presence of narrow $\rho_{T 8}$ 's and the destructive interference between the gluon and coloron - even though the flavor-universal coloron has a mass of 3-4.5 TeV! As in the first benchmark, none of the individual technirho resonances can be excluded with Run I data. The case $M_{\rho_{22}}=0.4 \mathrm{TeV}$ corresponds to the $M_{V_{8}} \tan \theta_{3}=0.84 \mathrm{TeV}$ limit set from the D $\varnothing$ dijet data by Bertram and 
Simmons [31] without including the $\rho_{T 8}$ 's.

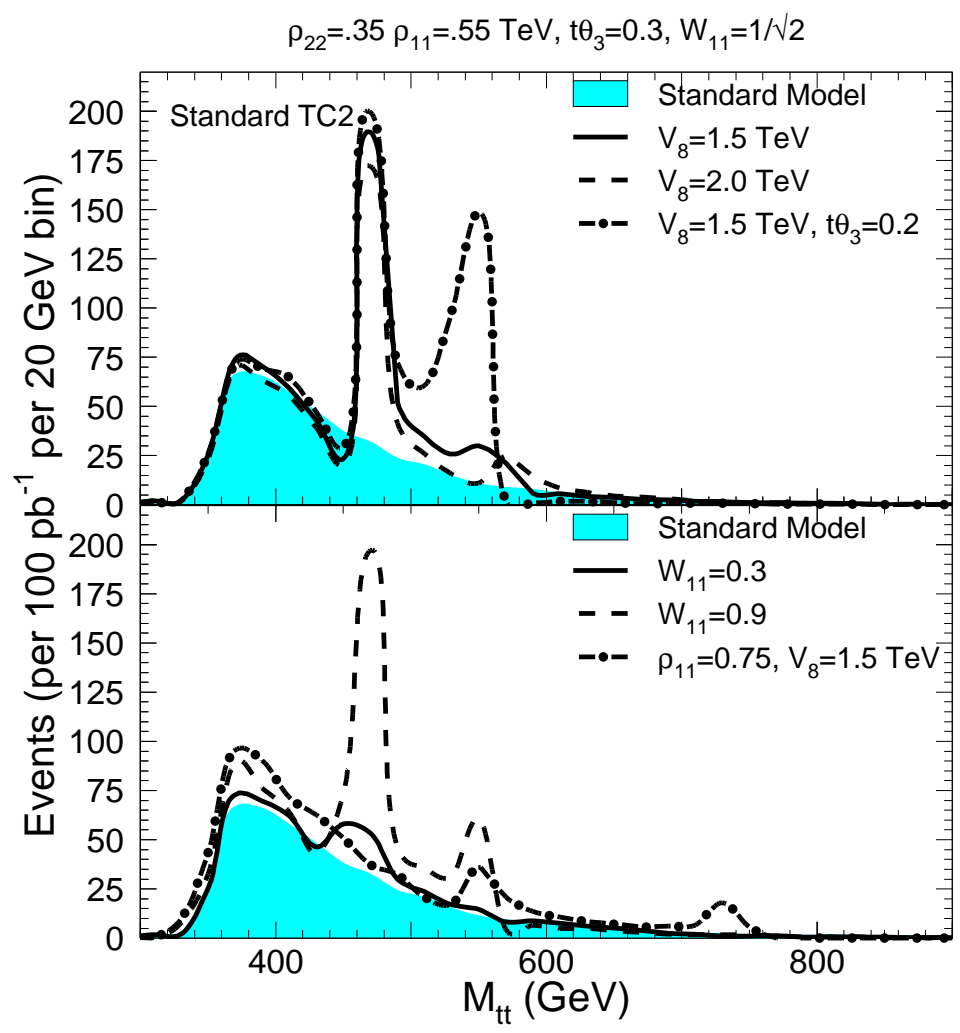

Figure 3: Similar to Fig. 1, but showing the variation with coloron mass.

\section{Benchmark III: Standard TC2- $\mathcal{M}_{\bar{t} t}$ with various $M_{V_{8}}$}

This benchmark demonstrates the variation with the coloron mass in standard TC2. The technirhos are fixed at the mass values of $M_{\rho_{22}}=.35 \mathrm{TeV}$, $M_{\rho_{11}}=.55 \mathrm{TeV}$, and $M_{\rho_{12,21}}=.45 \mathrm{TeV}$. This is specifically chosen so that the $\rho_{22}$ is below the $\bar{t} t$ threshold; the resultant peak in $\mathcal{M}_{\bar{b} b}$ (not shown here) is consistent with the Run I limits discussed earlier [19]. The results are shown in Fig. 3. For these choices of parameters, narrow resonances appear near $M_{\rho_{11}}$ and $M_{\rho_{21}}$. The appearance of two significant peaks for a lower value of $\tan \theta_{3}=0.2$ (upper panel, dash-dot) may be excludable by a multi- 
resonance analysis, but is presently allowed by the Run I data. A promising model-line for this benchmark is to vary the technirho mixing through $W$ and $\tan \theta_{3}$ with all other masses fixed. For extreme values of the coloron mass, this will become similar to Benchmarks V and VI described later.

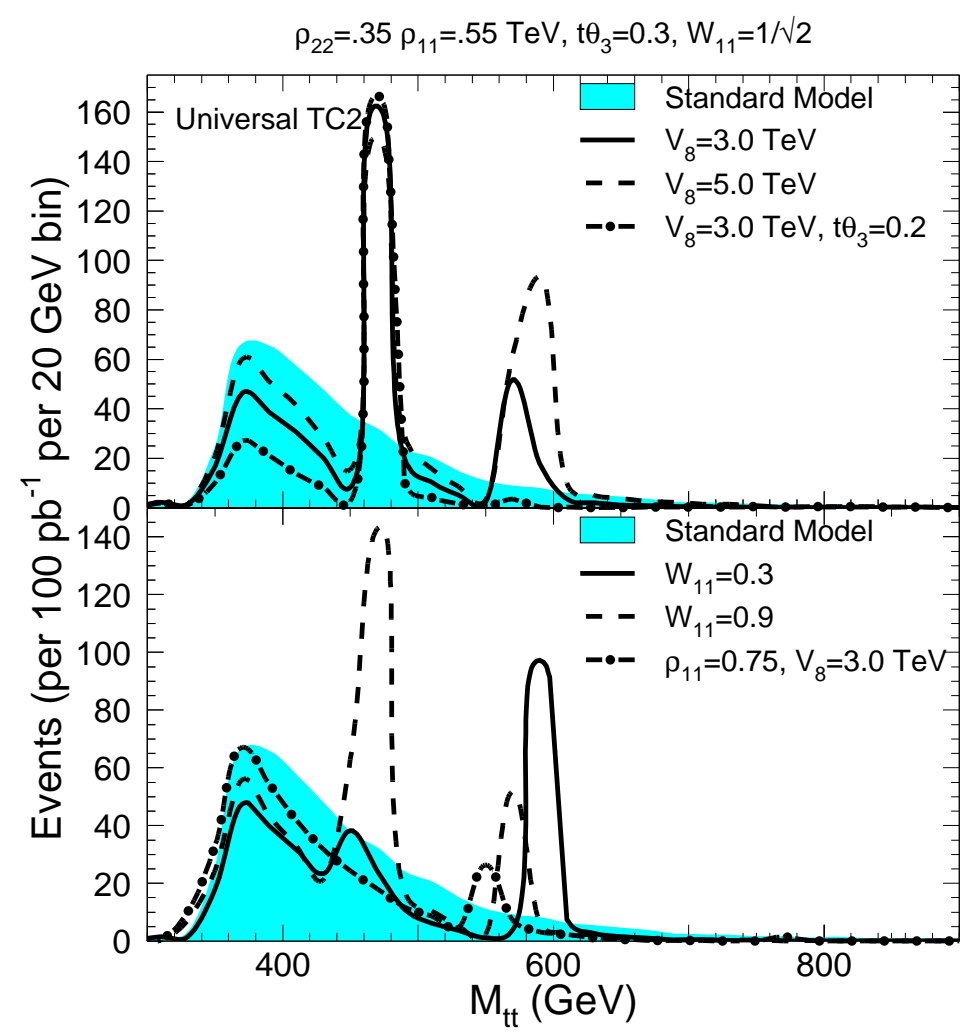

Figure 4: Similar to Fig. 2, but showing the variation with coloron mass.

Benchmark IV: Flavor-Universal TC2- $\mathcal{M}_{\bar{t} t}$ with various $M_{V_{8}}$

The parameter choices here are similar to that of Benchmark III for standard TC2, except that the colorons are heavier. The results are shown in Fig. 4. As in Benchmark III, there are narrow resonances, but destructive rather than constructive gluon-coloron interference. The standard model invariant mass distribution is greatly distorted, except for the case of a heavier 
$\rho_{11}$ (lower panel, dash-dot). The coloron mass $M_{V_{8}}=3 \mathrm{TeV}$ in this case is consistent with the limit obtained by Bertram and Simmons.

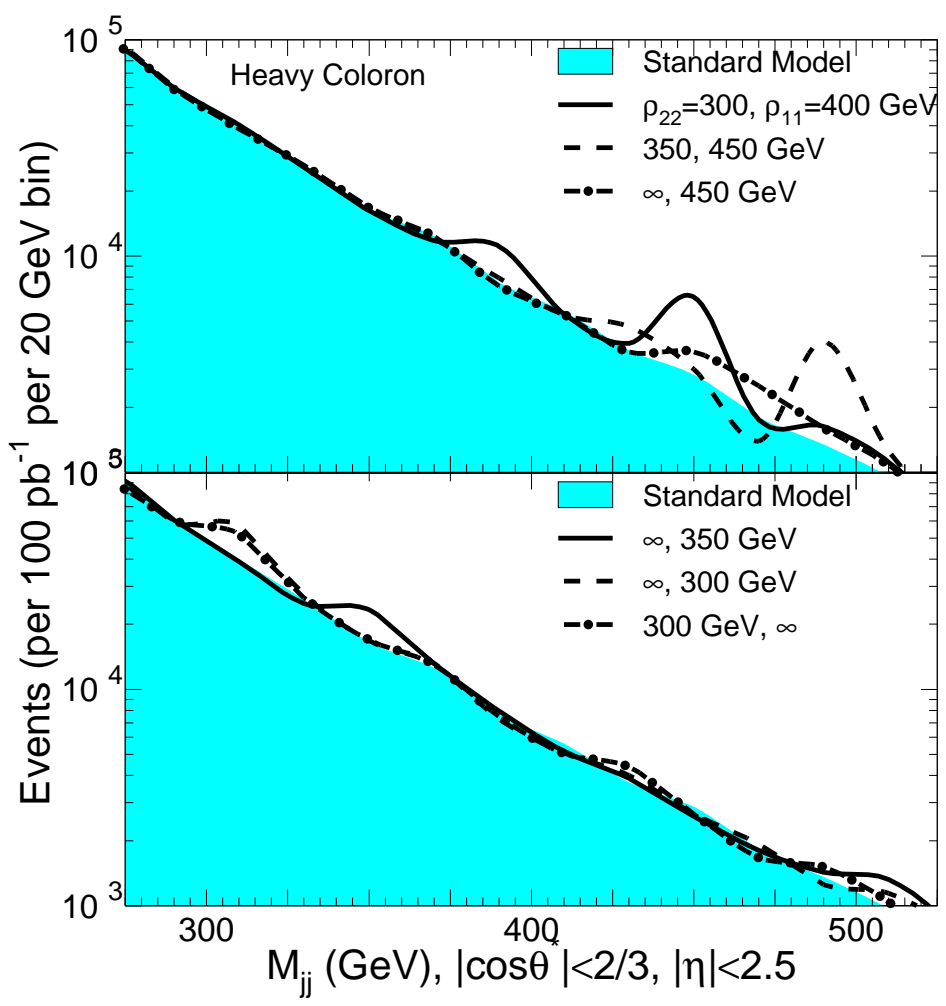

Figure 5: Dijet Invariant mass distributions compared to the standard model distribution for flavor universal TC2 with a heavy coloron, but light technirhos.

\section{Benchmark V-Universal TC2 jets with large $M_{V_{8}}$}

Our last two benchmarks illustrate the effect of flavor-universal TC2 on light-parton dijet mass distributions. For the first, the coloron is taken heavy, and we focus on the lighter technirho resonances. This corresponds to the CDF search in Run I for a single (pre-TC2) color octet technirho [18]. Dijets in this study were required to have $|\cos \theta|<2 / 3$ and $|\eta|<2.5$ for the cms 
scattering angle and rapidity. The search excluded a $\rho_{T 8}$ resonance in the mass range of $260-480 \mathrm{GeV}$. The standard model prediction is shown in Fig. 5 by the solid histogram. The first two curves are for technirho masses of $M_{\rho_{22,11}}=300,400 \mathrm{GeV}$ (solid) and $M_{\rho_{22,11}}=350,450 \mathrm{GeV}$ (dashes). As before, $M_{\rho_{12,21}}$ is taken as the average of these. Significant features appear in the invariant mass distribution, but shifted from the input $M_{\rho_{i j}}$ because of mixing effects in the $6 \times 6$ propagator matrix. The $300 \mathrm{GeV} \rho_{22}$ sits on too much background to be visible, i.e., in this model it could not be excluded by the CDF search in Run I. The last four curves treat the case of only one light technirho and, as expected, they exhibit resonant structure quite near the input mass. A promising model-line is to start with nearly equal values for $M_{\rho_{22}}$ and $M_{\rho_{11}}$, and slowly increase $M_{\rho_{11}}$ until the single technirho limit is reached. Also, the sensitivity to mass splitting and the mixing parameter $M_{8}^{\prime}$ should be considered.

Benchmark VI-Universal TC2 jets with moderate $M_{V_{8}}$

The last benchmark studies the effect of a coloron from flavor-universal TC2 on the dijet invariant mass distributions. In Run I, CDF [18] and DØ [43] excluded a flavor-universal coloron with masses less than $759-980$ $\mathrm{GeV}$, depending on $\tan \theta_{3}$. We have chosen $M_{\rho_{11,22}}=450,550 \mathrm{GeV}$, but they have little effect on the distribution. The main parameters are the coloron mass and coupling. For $M_{V_{8}}=1.5 \mathrm{TeV}$ and $\tan \theta_{3}=0.3$ (upper panel, solid), there is a significant enhancement over the standard model distribution. However, for $M_{V_{8}}=2 \mathrm{TeV}$ (dashes) and $2.5 \mathrm{TeV}$ (dash-dot), there is little distortion. Note that, for ordinary dijet production, the effect of a flavor universal coloron below resonance is to increase the cross section (see also [42]). This is because of the dominance of $\hat{t}$ channel coloron exchange in the QCD subprocesses, which cannot interfere destructively.

We have presented numerical results for Tevatron Run I to indicate, crudely, the range of TC2 parameters allowed by the data. Much more systematic studies need to be carried out for the parameter ranges allowed by existing data and the reach anticipated for future data. These studies should include detector effects, efficiencies, etc. The expected improvement from Tevatron Run II will be statistical, resulting from the increased beam luminosity and partonic luminosity at larger $x$. Since the standard model $\bar{t} t$ cross section increases similarly, the relative importance of colorons or technirhos is basically unchanged. To make one more contact with Run I studies, we show in Fig. 7 the expected contribution of a single, narrow technirho to 


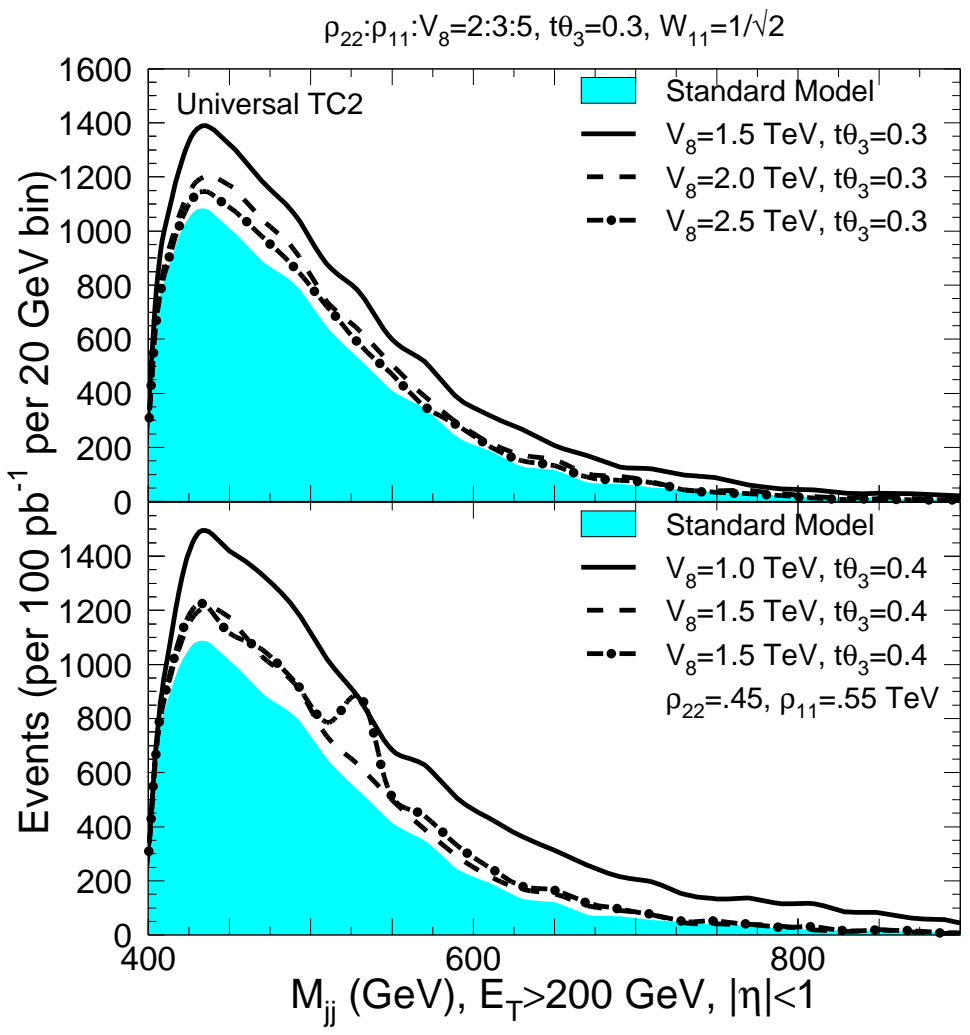

Figure 6: Similar to Fig. 5, except considering light colorons.

the $\bar{t} t$ distribution (simulated by making the other technihadrons and coloron very heavy). This case is unrepresentative of any TC2 model.

Finally, in our examples, we focussed on TC2 effects on standard model final states. The decays $\rho_{T 8} \rightarrow g \pi_{T}^{0 \prime}$ and $g \pi_{T 8}$ were included when calculating the $\rho_{T 8}$ width, but the direct production of $\rho_{T 8}$ followed by the subsequent decay $\pi_{T}$ was not. A direct search for $\rho_{T 8} \rightarrow g \pi_{T}^{0 \prime} \rightarrow g \bar{b} b$ would be challenging. The default decay of $\pi_{T 8}$, motivated by certain TC2 models, is to two gluons. This is probably impossible to isolate above backgrounds. Nevertheless, a search for $\pi_{T 8} \rightarrow \bar{b} b$ (or even $\bar{t} t$ ) would be worthwhile. 


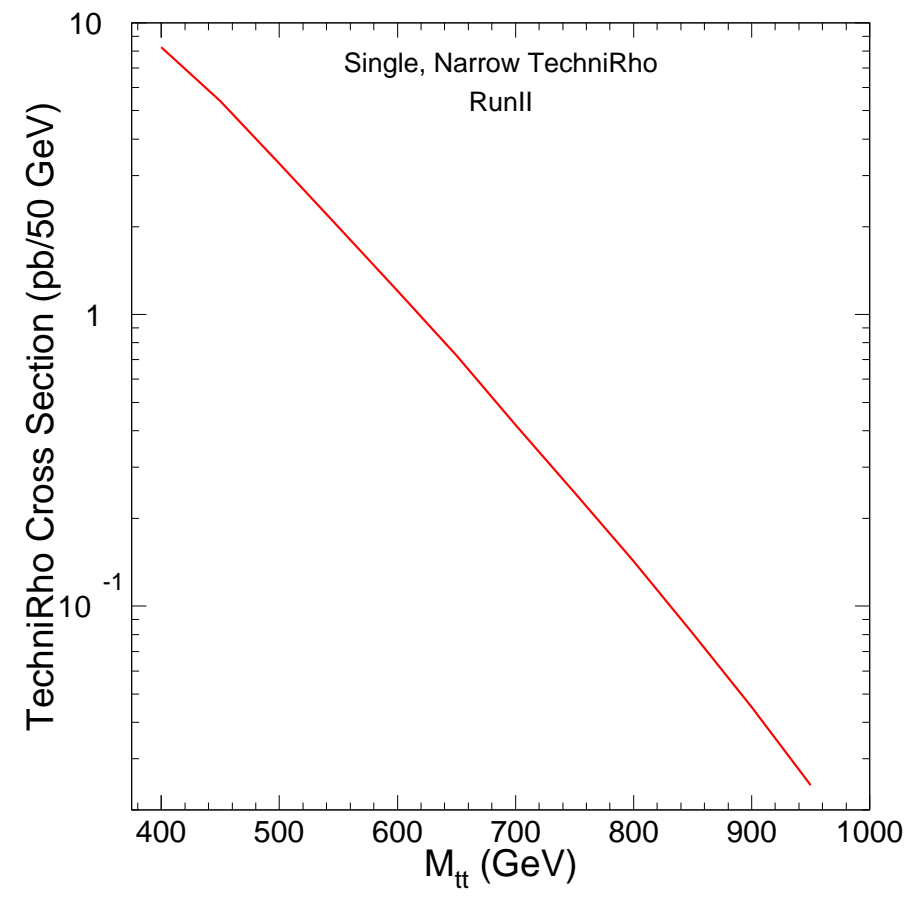

Figure 7: The additional cross section (in pb) from a narrow technirho resonance in a $50 \mathrm{GeV}$ bin of the $\bar{t} t$ distribution for the Tevatron Run II.

\section{Discussion and Conclusions}

In this paper, we improved and extended the technicolor straw man model for testing low-scale technicolor, i.e., the notion that dynamical electroweak symmetry breaking requires a large number of electroweak doublets of technifermion and, therefore, that the technicolor energy scale may be only a few hundred $\mathrm{GeV}$. The improvements were made to the color-singlet sector of the TCSM, and are largely applicable to $e^{+} e^{-}$colliders operating below the narrow $\rho_{T}$ and $\omega_{T}$ resonances expected at several hundred $\mathrm{GeV}$. The principal signals here are $\pi_{T}$ production - either in association with a $\gamma, W^{ \pm}$, or $Z^{0}$, or in pairs - or a narrow $\ell^{+} \ell^{-}$resonance. 
We extended the TCSM by including a color-nonsinglet sector. This is based on models of topcolor-assisted technicolor in which topcolor is broken by technifermion condensates. This approach, summarized in Section 1, is still the only fully-described dynamical scheme for generating the top quark's mass. It, too, requires low-scale technicolor. Although the naturalness of TC2 - the requirement that topcolor gauge boson masses, $M_{V_{8}}$ and $M_{Z^{\prime}}$, not be much larger than $1 \mathrm{TeV}$-is challenged by $B$-meson mixing measurements, there is enough room to evade those constraints that other, independent tests of TC2 are needed. Hadron collider experiments are particularly incisive. There, production of ordinary jet and $\bar{t} t$ and $\bar{b} b$ pairs may reveal color octet $\rho_{T 8}$ and $V_{8}$ resonances, the latter for subprocess energies well below $M_{V_{8}}$. We considered both variants of TC2, the standard version proposed by Hill in which the coloron couples strongly only to top and bottom quarks, and the flavor-universal TC2 advanced by Simmons and her collaborators. For a large and probable range of TCSM parameter space, the effects of these two variants on $\bar{t} t$ production are striking, and strikingly different.

All of the color-singlet and nonsinglet processes discussed in this paper are now included in the event generator PYTHIA. This should provide many happy hours of detailed studies by experimental collaborations at the Tevatron and the LHC, as well as refined reanalysis of data from LEP and opportunities for those yearning for studies of nonsupersymmetric physics in the $\mathrm{TeV}$ region of a linear $e^{+} e^{-}$collider. An application to LEP physics was presented in Ref. [3]. To illuminate our discussion for hadron colliders, we considered several benchmark parameter sets and studied their effect on observables for Tevatron Run I conditions. Our parton-level studies with PYThiA are qualitative. A quantitative discussion of jet or heavy quark observables requires detailed simulations, including features of real detectors and running conditions. We are encouraged that, where a comparison is possible, our numerical results are in reasonably good agreement with published Run I limits on colorons and technirhos.

Top quark pair production at the Tevatron is an ideal probe for topcolor, TC2 and other new dynamics associated with producing $m_{t}$, since the final state is distinctive and it is produced through strong interactions dominated by valence quark annihilation. There is great sensitivity to the coloron even far below resonance. Furthermore, signals for the technirhos, which are expected to be lighter than the coloron, are sensitive to the coloron mass through mixing effects. For standard TC2, the coloron interferes 
constructively with the gluon in $s$-channel processes, and enhances the production cross section, fairly independently of the $S U(3)$ mixing parameter, $\tan \theta_{3}$. Without including relatively light $\rho_{T 8}$, the Run I lower bound on the standard TC2 coloron mass is $M_{V_{8}}>1-2 \mathrm{TeV}$. On the other hand, there is a significant reduction of the cross section for flavor-universal TC2. This effect is further enhanced by the direct appearance of $\tan \theta_{3}$ in the effective couplings between light and heavy quarks. The Run I coloron bound is $M_{V_{8}}>3-4 \mathrm{TeV}$, depending on $\tan \theta_{3}$. Since $\rho_{T 8}$ are expected to be lighter than the coloron, their appearance as narrow peaks in heavy or light quark pair production places more stringent bounds on the coloron mass in either model.

\section{Acknowledgements}

The model described here will be available in PYTHIA v6.211.

We are grateful for inspiration, advice, and encouragement from Guennadi Borissov, Sekhar Chivukula, Estia Eichten, Robert Harris, Chris Hill, Andrei Kounine, Meenakshi Narain, Stephen Parke, Francois Richard, Markus Schumacher, Elizabeth Simmons, John Womersley and other members of the "Strong Dynamics for Run II Workshop" at Fermilab. KL's research was supported in part by the Fermilab Theory Group Frontier Fellowship program and by the Department of Energy under Grant No. DE-FG02-91ER40676. FNAL is operated by Universities Research Association Inc., under contract DE-AC02-76CH03000. 


\section{Appendix A: Default Values for Parameters}

The suggested default values of the parameters used in this note are listed in Table 3.

\begin{tabular}{|c|c|c|c|}
\hline Parameter & Default Value & Parameter & Default Value \\
\hline \hline$N_{T C}$ & 4 & $\alpha_{\rho_{T}}\left(N_{T C} / 3\right)$ & 2.91 \\
$\sin \chi$ & $\frac{1}{3}$ & $\sin \chi^{\prime}$ & $1 / \sqrt{6}$ \\
$Q_{U}$ & $\frac{4}{3}$ & $Q_{D}=Q_{U}-1$ & $\frac{1}{3}$ \\
$C_{1 \tau, c, b}$ & 1 & $C_{1 t}$ & $m_{b} / m_{t}$ \\
$C_{1 g}^{2}$ & $\frac{4}{3}$ & $\mid \epsilon_{\rho \omega}$ & 0.05 \\
\hline$F_{T}=F_{\pi} \sin \chi$ & $82 \mathrm{GeV}$ & $M_{V, A}$ & $200 \mathrm{GeV}$ \\
$M_{\rho_{T}^{ \pm}, \omega_{T}, \rho_{T}^{0}}$ & $210 \mathrm{GeV}$ & $M_{\pi_{T}^{ \pm}, \pi_{T}^{0}, \pi_{T}^{0 \prime}}$ & $110 \mathrm{GeV}$ \\
\hline $\sin \chi^{\prime \prime}$ & $1 / \sqrt{2}$ & $\tan \theta_{3}$ & $\sqrt{0.08}$ \\
$C_{8 s, c, b, t}$ & 0 & $C_{8 g}^{2}$ & $\frac{5}{3}$ \\
$M_{\pi_{T 8}}^{2}$ & $250 \mathrm{GeV}$ & $M_{V_{8}}$ & $500 \mathrm{GeV}$ \\
$M_{\rho_{11}}$ & $400 \mathrm{GeV}$ & $M_{\rho_{22}}$ & $300 \mathrm{GeV}$ \\
$M_{\rho_{12,12^{\prime}}}$ & $350 \mathrm{GeV}$ & $M_{8}, M_{8}^{\prime}$ & $250 \mathrm{GeV}$ \\
$W_{L 11}^{U, D}$ & $1 / \sqrt{2}$ & $\phi_{U, D}$ & 0 \\
\hline \hline
\end{tabular}

Table 3: Default values for parameters in the Technicolor Straw Man Model. As in Refs. $[1,15]$, the technipion decay constant is $F_{T}=F_{\pi} \sin \chi$ with a default value of $82 \mathrm{GeV}$, and the $\rho_{T} \rightarrow \pi_{T} \pi_{T}$ coupling is $\alpha_{\rho_{T}}=2.91\left(3 / N_{T C}\right)$. The default model is standard TC2 [13]. We have set $W_{R}^{U, D}=1$ and used Eq. (5) for the matrix elements $W_{L i j}^{U, D}$. 


\section{References}

[1] K. Lane, Phys. Rev. D60, 075007 (1999) [hep-ph/9903369].

[2] L. Randall and E. H. Simmons, Nucl. Phys. B380, 3 (1992);

G. Rupak and E. H. Simmons, Phys. Lett. B362, 155 (1995) [hep-ph/9507438];

K. R. Lynch and E. H. Simmons, Phys. Rev. D64, 035008 (2001) [hep-ph/0012256].

[3] K. Lane, et al., Phys. Rev. D66, 015001 (2002) [hep-ph/0203065].

[4] S. Mrenna, Phys. Lett. B 461, 352 (1999) [hep-ph/9907201].

[5] The L3 Collaboration, L3 Physics Note 2428.

[6] J. Abdallah, et al., The DELPHI Collaboration, Eur. Phys. J. C22, 17 (2001) [hep-ex/0110056].

[7] The OPAL Collaboration, Searches for Technicolor with the OPAL Detector in $e^{+} e^{-}$Collisions at the Highest LEP Energies, (2001), OPAL Physics Note PN485.

[8] T. Sjöstrand, et al., Comput. Phys. Commun. 135, 238, (2001) [hep-ph/0010017];

T. Sjöstrand, L. Lonnblad, and S. Mrenna, (2001) [hep-ph/0108264].

[9] S. Weinberg, Phys. Rev. D19, 1277 (1979);

L. Susskind, Phys. Rev. D20, 2619 (1979).

[10] E. Eichten and K. Lane, Phys. Lett. B90, 125 (1980).

[11] B. Holdom, Phys. Rev. D24, 1441 (1981);

Phys. Lett. 150B, 301 (1985);

T. Appelquist, D. Karabali and L. C. R. Wijewardhana, Phys. Rev. Lett. 57, 957 (1986);

T. Appelquist and L. C. R. Wijewardhana, Phys. Rev. D36, 568 (1987);

K. Yamawaki, M. Bando and K. Matumoto, Phys. Rev. Lett. 56, 1335 (1986);

T. Akiba and T. Yanagida, Phys. Lett. 169B, 432 (1986). 
[12] C. T. Hill, Phys. Lett. 266B, 419 (1991);

S. P. Martin, Phys. Rev. D45, 4283 (1992);

ibid D46, 2197 (1992); Nucl. Phys. B398, 359 (1993);

M. Lindner and D. Ross, Nucl. Phys. B370, 30 (1992);

R. Bönisch, Phys. Lett. 268B, 394 (1991);

C. T. Hill, D. Kennedy, T. Onogi, H. L. Yu, Phys. Rev. D47, 2940 (1993).

[13] C. T. Hill, Phys. Lett. 345B, 483 (1995) [hep-ph/941142].

[14] K. Lane and E. Eichten, Phys. Lett. B222, 274 (1989).

[15] E. Eichten and K. Lane, Phys. Lett. B388, 803 (1996) [hep-ph/9607213];

E. Eichten, K. Lane and J. Womersley, Phys. Lett. B405, 305 (1997) [hep-ph/9704455];

E. Eichten, K. Lane and J. Womersley, Phys. Rev. Lett. 80, 5489 (1998) [hep-ph/9802368].

[16] T. Affolder, et al., The CDF Collaboration, Phys. Rev. Lett. 84, 1110 (2000).

[17] F. Abe, et al., The CDF Collaboration, Phys. Rev. Lett. 83, 3124 (1999) [hep-ex/9810031].

[18] F. Abe, et al., The CDF Collaboration, Phys. Rev. D55, 5263 (1997) [hep-ex/9702004].

[19] F. Abe et al., The CDF Collaboration, Phys. Rev. Lett. 82, 2038 (1999) [hep-ex/9809022].

[20] F. Abe, et al., The CDF Collaboration, Phys. Rev. Lett. 82, 3206 (1999).

[21] Atlas Physics Technical Design Report, Chapter 21 (1999), available at http://atlasinfo. cern. ch/Atlas/GROUPS/PHYSICS/TDR/access.html.

[22] K. Lane, Technicolor Production and Decay Rates in the Technicolor Straw Man Model, [hep-ph/9903372], Boston University Preprint BUHEP-99-5, March 1999. Note that the present paper supersedes the production, but not the decay, rates compiled in this paper. 
[23] K. Lane, Phys. Rev. D54, 2204 (1996) [hep-ph/9602221];

K. Lane, Phys. Lett. B433, 96 (1998) [hep-ph/9805254].

[24] E. Eichten, I. Hinchliffe, K. Lane and C. Quigg, Rev. Mod. Phys 56, 579 (1984); Phys. Rev. DD34, 1547 (1986).

[25] K. Lane and M. V. Ramana, Phys. Rev. D44, 2678 (1991).

[26] R. S. Chivukula and J. Terning, Phys. Lett. B385, 209 (1996) [hep-ph/9606233].

[27] T. Rador, Phys. Rev. D59, 095012 (1999) [hep-ph/9810252].

[28] R. S. Chivukula, A. G. Cohen and E. H. Simmons, Phys. Lett. B380, 92 (1996) [hep-ph/9603311];

M. Popovic and E. H. Simmons, Phys. Rev. D58, 095007 (1998) [hep-ph/9806287].

[29] G. Burdman, K. Lane, and T. Rador, Phys. Lett. B514, 41 (2001) [hep-ph/0012073].

[30] E. H. Simmons, Phys. Lett. 526, 365 (2002) [hep-ph/0111032].

[31] I. Bertram and E. H. Simmons, Phys. Lett. B443, 347 (1998) [hep-ph/9809472].

[32] K. Lane, T. Rador and E. Eichten, Phys. Rev. D62, 015005 (2000) [hep-ph/0001056];

K. Lane, Strong and Weak CP Violation in Technicolor, Invited talk at the Eighth International Symposium on Particles, Strings and Cosmology - PASCOS 2001, University of North Carolina, Chapel Hill, NC, April 10-15, 2001 [hep-ph/0106328].

[33] R. S. Chivukula, B. Dobrescu and J. Terning, Phys. Lett. B353, 289 (1995) [hep-ph/9503203].

[34] K. Lane and E. Eichten, Phys. Lett. B352, 382 (1995) [hep-ph/9503433].

[35] E. Eichten and K. Lane, Phys. Lett. B327, 129 (1994) [hep-ph/9401236]. 
[36] A. Zerwekh and R. Rosenfeld, Phys. Lett. B503, 325 (2001) [hep-ph/0103159].

[37] R. S. Chivukula, A. Grant and E. H. Simmons, Phys. Lett. B521, 239 (2001) [hep-ph/0109029].

[38] C. T. Hill and S. Parke, Phys. Rev. D49, 4454 (1994) [hep-ph/9312324].

[39] K. D. Lane, Phys. Rev. D52, 1546 (1995) [hep-ph/9501260].

[40] T. Affolder et al., The CDF Collaboration, Phys. Rev. Lett. 85, 2062 (2000) [hep-ex/0003005].

[41] B. Abbott et al., The DØ Collaboration, Phys. Rev. D58, 052001 (1998) [hep-ex/9801025].

[42] E. H. Simmons, Phys. Rev. D 55, 1678 (1997) [hep-ph/9608269].

[43] B. Abbott et al., The DØ Collaboration, [hep-ex/9809009]. 